\title{
54. DATA REPORT: MASS ACCUMULATION RATE CALCULATIONS AND LABORATORY DETERMINATIONS OF CALCIUM CARBONATE AND EOLIAN MATERIAL IN NEOGENE SEDIMENTS FROM THE MARSHALL ISLANDS, SITES 871, 872, AND 873¹
}

\author{
Frank R. Rack, ${ }^{2}$ Horst W. Bohrmann, ${ }^{3}$ and Peter R.N. Hobbs ${ }^{4}$
}

\begin{abstract}
The pelagic sediment caps on guyots in the Marshall Islands record the accumulation of biogenic and lithogenic (eolian) particles in the northwestern region of the tropical Pacific Ocean throughout the Neogene. These guyots are located in a remote geographic region, far from continental sources of eolian input. Nonetheless, the fine-grained lithogenic component of the bulk sediment should record changes in the atmospheric transport of eolian grains to these sites as the Marshall Island guyots were moved across the equatorial region during the Neogene.

The weight percentages of calcium carbonate, noncarbonate, and lithogenic (eolian) sedimentary components have been determined from samples in the upper $50 \mathrm{~m}$ of pelagic sediment at Ocean Drilling Program Sites 871, 872 and 873 . For each site, two versions of modified shipboard biostratigraphic age models have been used to calculate mass accumulation rates for the bulk sediment and for each of the primary sedimentary components. These data are used to demonstrate the correspondence between temporal changes in biogenous and eolian sediment accumulation rates and the fluctuations in mass sediment physical properties observed in the upper $50 \mathrm{~m}$ of the pelagic caps on these guyots. These comparisons may help identify the effects of climatically induced changes in the position and intensity of equatorial wind systems and the resulting variations in ocean current effects (erosion and winnowing) on the pelagic cap at each of these three sites as they moved northward during the Neogene.
\end{abstract}

\section{INTRODUCTION}

The Marshall Islands are located in the northwestern region of the tropical Pacific Ocean (see site map preceding title page). The drill sites located on Limalok, Lo-En, and Wodejebato guyots (Sites 871877 ) have been transported northwestwardly, across the equatorial system of atmospheric wind belts and ocean currents to their presentday locations, by the motion of the Pacific Plate during the Neogene.

The guyots in the Marshall Islands region have pelagic caps that range in thickness from virtually 0 to $150 \mathrm{~m}$. The thickness of an individual pelagic cap appears to be strongly correlated with both the depth of the guyot (related to the length of the depositional history) and the size of the guyot (less erosion occurs near the perimeter of the summit; van Waasbergen and Winterer, 1993). A southeastto-northwest transect of three different Marshall Island guyots was drilled during Ocean Drilling Program (ODP) Leg 144. The drill sites on these three guyots are geographically distributed along different chains of seamount lineations (Sitè 871, Ratak Chain; Site 873, Ralik Chain; and Site 872, furthest westward; see Fig. 1).

The objectives of this study were to determine the abundances (weight percentage) and accumulation rates of the calcium carbonate, noncarbonate, and lithogenic ("eolian") components of the total sediment flux to the pelagic caps of three guyots in the Marshall Islands (Limalok, Lo-En, and Wodejebato guyots) from the early middle Miocene to the Holocene, and to compare the temporal variations in these sedimentary fluxes to changes in mass physical properties.

\footnotetext{
'Haggerty, J.A., Premoli Silva, L., Rack, F., and McNutt, M.K. (Eds.), 1995. Proc. $O D P$, Sci. Results, 144: College Station, TX (Ocean Drilling Program).

${ }^{2}$ Ocean Mapping Group, Department of Geodesy and Geomatics Engineering, University of New Brunswick. Fredericton, New Brunswick, Canada E3B 5A3.

GEOMAR, Forschunszentrum fuer Marine Geowissenschaften, Wischofstrasse 1-3, D-24148 Kiel, Federal Republic of Germany.

${ }^{4}$ British Geological Survey, Engineering Geology and Geophysics Group, Keyworth, Nottingham NG125GG, United Kingdom.
}

\section{BACKGROUND AND OBJECTIVES}

\section{Geologic Setting}

\section{Site 871}

Site 871 is located at $5^{\circ} 33.43^{\prime} \mathrm{N}, 172^{\circ} 20.66^{\prime} \mathrm{E}$ in $1255 \mathrm{~m}$ water depth, in the south central portion of Limalok Guyot, in the Ratak Chain of the southern Marshall Islands (Fig. 1). This site is the southernmost of the north-south transect of sites drilled by Leg 144 and is considered to be the youngest of the guyots in the Marshall Island region (Lincoln et al., 1993).

The sediment in the pelagic cap on Limalok Guyot at Site 871 (0-133.7 $\mathrm{m}$ below seafloor [mbsf]) consists of foraminifer nannofossil ooze and foraminifer ooze of Pleistocene to early Miocene age. The pelagic cap is divided into two subunits at $26.5 \mathrm{mbsf}$, where light gray nannofossil foraminifer ooze of Pleistocene to latest Miocene age above changes to white homogeneous foraminifer ooze of middle to early Miocene age below. The transition between the two subunits is marked by a hiatus that spans the late middle to latest Miocene. The sediment in the lower portion of the pelagic cap is well-sorted and winnowed, with a medium sand texture. A prominent disconformity, spanning the late middle Miocene through most of the late Miocene (approximately 6 m.y.), marks a major change in sediment accumulation on this guyot (Premoli Silva, Haggerty, Rack, et al., 1993).

\section{Site 872}

Site 872 is located at $10^{\circ} 05.85^{\prime} \mathrm{N}, 162^{\circ} 51.96^{\prime} \mathrm{E}$ in $1084 \mathrm{~m}$ water depth, on the central portion of Lo-En Guyot (Fig. 1). This site is located approximately $148.2 \mathrm{~km}$ southeast of Anewetak Atoll in the northern Marshall Islands. The long-range atmospheric transport of soil dust from Asia to the tropical oceanic region near Anewetak Atoll has been the subject of several recent investigations by groups of atmospheric chemists (Duce et al., 1980, 1983; Ueumatsu et al., 1983; Arimoto et al., 1985) and paleoceanographers (Janecek and Rea, 1983).

The pelagic cap on Lo-En Guyot consists of an upper lithologic subunit of white to very pale brown nannofossil foraminifer ooze and 


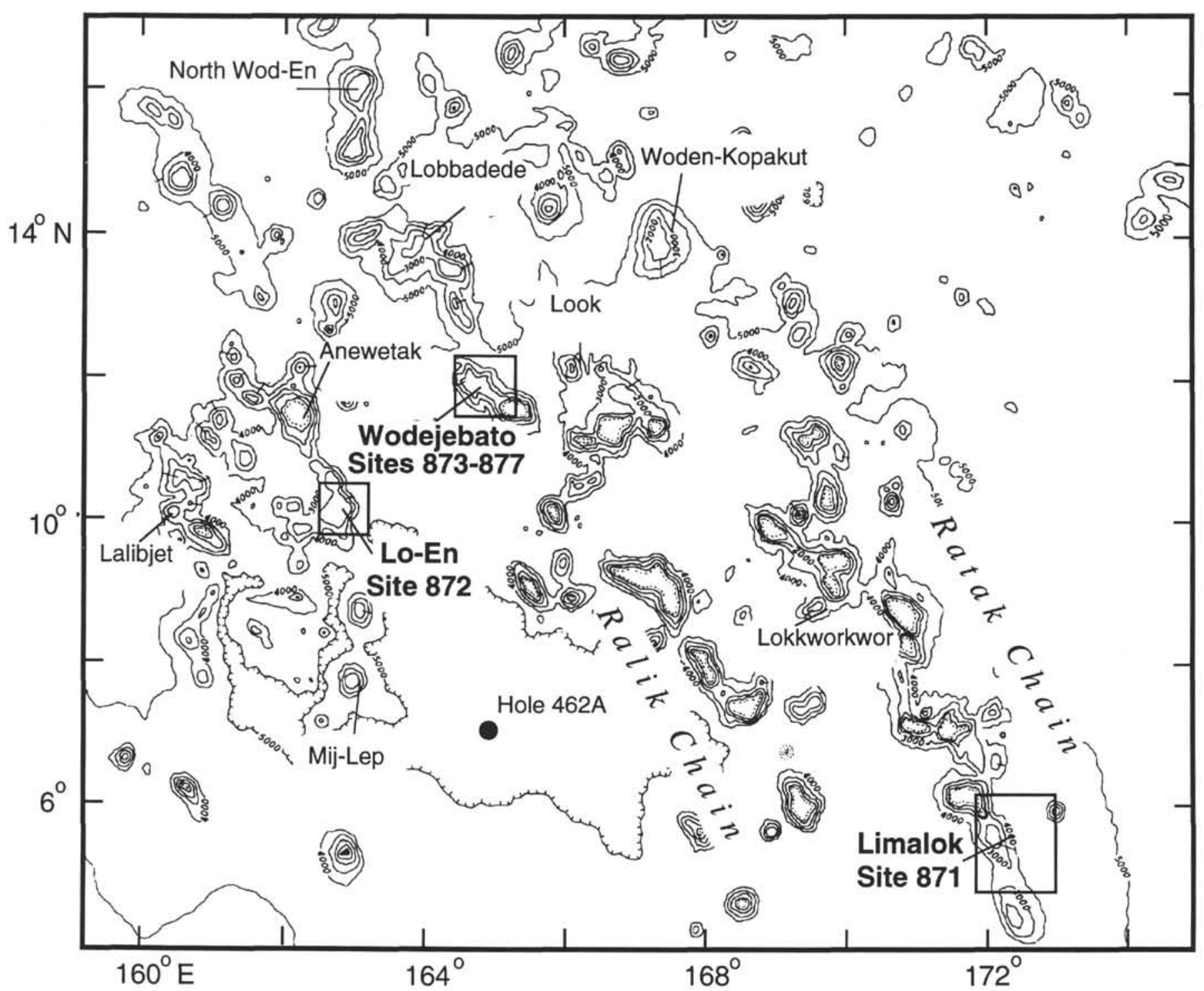

Figure 1. Location map of atolls and guyots in the Marshall Islands. Boxes enclose the location of ODP sites drilled on Limalok (871), Lo-En (872), and Wodejebato (873-877) guyots (Premoli Silva, Haggerty, Rack, et al., 1993).

intercalated foraminifer ooze of Pleistocene to late Miocene age (30-32 m thick), and a lower subunit of very pale brown homogeneous foraminifer ooze, which is well sorted and winnowed, with a medium sand texture and of a late Miocene to late Oligocene age. The transition between the two subunits is marked by a physically mixed and incomplete interval of nannofloras and faunas of different zones, which spans the late middle to early late Miocene, overlain by a disconformity (Premoli Silva, Haggerty, Rack, et al., 1993).

\section{Site 873}

Site 873 is situated on the south central summit of Wodejebato Guyot, at $11^{\circ} 53.84^{\prime} \mathrm{N}, 164^{\circ} 55.20^{\prime} \mathrm{E}$, in the Ralik Chain of the northern Marshall Islands (Fig. 1). The pelagic cap on Wodejebato Guyot consists of an upper lithologic subunit of light gray nannofossil foraminifer ooze of Pleistocene to latest Miocene age; whereas, the lower subunit is composed of a well-sorted and winnowed, very pale brown, mainly homogeneous foraminifer ooze with medium sand texture, and of middle to early Miocene age. The transition between the two subunits is marked by a disconformity spanning most of the late and late middle Miocene (Premoli Silva, Haggerty, Rack, et al., 1993).

\section{Past and Future Research Questions}

A detailed evaluation of the similarities and differences in the accumulation of pelagic sediment on the summits of Limalok, Lo-En, and Wodejebato guyots should provide insight into the evolution of the equatorial ocean-atmosphere system during the Neogene.

Changes in physical properties (bulk density, porosity, water content) of the pelagic cap sediments reflect changes in the depositional environment (e.g., winnowing, erosion) during accumulation of these biogenic oozes and changes in sedimentary inputs (e.g., relative abundance of foraminifers vs. nannofossils, and eolian fluxes). An improved understanding of the onset, duration, and continuity (both laterally and temporally) of the major hiatus(es) in sediment accumulation within the pelagic cap at each of these sites, especially during the late-middle Miocene to late Miocene (major unconformity at Sites 871 and 873 , less at Site 872), can be used to identify past changes in the intensity and relative latitudinal position of the various equatorial paleocurrents. These currents, which are influenced by changes in atmospheric wind belts, have surely influenced, and may have controlled, the depositional environments on these guyots lying at intermediate water depths. 


\section{METHODS}

The sediment samples used in this study were collected during Leg 144 on board the JOIDES Resolution. Samples with a volume of approximately $10 \mathrm{~cm}^{3}$ were collected at a regular sample spacing of $50-75 \mathrm{~cm}$, in cores from the upper $60 \mathrm{mbsf}$ at Holes $871 \mathrm{~A}$, $872 \mathrm{~A}, 872 \mathrm{C}$, and $873 \mathrm{~B}$. The samples were sealed in bags during the cruise. Onshore, they were freeze-dried and weighed immediately before processing.

\section{Carbonate Analyses}

The inorganic carbon content of each sample was determined using a Coulometrics carbon dioxide coulometer. Freeze-dried samples weighing between 10 and $20 \mathrm{mg}$ were reacted in $2 \mathrm{~N} \mathrm{HCl}$ solution at $60^{\circ} \mathrm{C}$. Evolved carbon dioxide was titrated in a monoethanolamide solution with a coulometric indicator over a period of 5-20 min, depending on the carbonate reactivity. Calibration was performed using pure calcium carbonate as a standard. The percentage of carbonate was calculated from the inorganic carbon (IC) content, assuming that all carbonate was in the form of calcite: $\mathrm{CaCO}_{3}=\mathrm{IC} \times 8.332$.

Duplicate analyses of 30 samples indicate a reproducibility of $0.7 \%$ with this method. The percentage of noncarbonate material was determined by subtracting the weight percentage of calcium carbonate from $100 \%$.

\section{Procedures for Eolian Extractions}

Following the determination of inorganic carbon, the remaining portion of each sample from Holes 871A and 873B was weighed and then processed according to a lithogenic extraction procedure, as described in Rea and Janecek (1981) and in the appendix to Clemens and Prell (1990).

The fine-grained lithogenic ("eolian") component of the bulk sediment was isolated by a series of selective extractions to remove calcium carbonate, opaline silica, $\mathrm{Fe}$ and $\mathrm{Mn}$ oxides, and hydroxides and zeolites. The $<63-\mu \mathrm{m}$-sized material remaining after the completion of the extraction procedure was weighed to determine the "eolian" weight percentage of the total sample. The eolian fraction was dominated by clays and fine-grained quartz.

Because of the very low amounts of detrital minerals anticipated in these sediments from the western equatorial Pacific, pairs of successive samples were combined to conduct the extraction procedure. This process degraded the overall temporal resolution of the eolian flux determinations, but improved the accuracy of the analytical measurements.

\section{Accumulation Rate Calculations}

For each site, a slightly modified, "comprehensive" version of the biostratigraphic age model (see Premoli Silva, Haggerty, Rack, et al., 1993) was produced. An attempt was made to reduce, as much as possible, the temporal uncertainty created by the use of multiple and often conflicting biostratigraphic datums (e.g., first appearance [FAD] and last appearance [LAD] datums from both calcareous nannofossil and foraminifer species) in this model. A "simplified" age model, based on only a few commonly observed biostratigraphic datums, was generated for each site to minimize any extreme fluctuations in calculated linear sedimentation rates.

The simplified age model was based on five (foraminifer or nannofossil) datums found at two of the three sites (three datums were common to all three sites; see Premoli Silva, Haggerty, Rack, et al., 1993) and which avoided the intervals of major regional hiatuses in sediment accumulation. The five datums used to generate the simplified age model for each site were: (1) the LAD of $H$. sellii $(1.37 \mathrm{Ma})$, (2) the FAD of $P$. primalis (5.8 Ma), (3) the LAD of $G$. fohsi lobato (11.5 Ma), (4) the LAD of S. heteromorphous (14.4 Ma), and (5) the FAD of $S$. heteromorphous (17.10 Ma).
Because of the presence of a condensed late Miocene sequence at Site 873 , it was necessary to substitute the LAD of T. rugosus $(\sim 5.0$ Ma) for the FAD of $P$. primalis, and the LAD of $C$. floridanus (11.60 $\mathrm{Ma}$ ) for the LAD of $G$. fohsi lobata in the simplified age model.

The two age models (i.e., the comprehensive and simplified models) generated for each site were used to calculate successive linear sedimentation rates (LSR) relative to the midpoint depth (mbsf) of each biostratigraphic datum. At each site, this procedure was repeated to calculate linear sedimentation rates using both the complete and the simplified age models. Dry density values were determined for each sample using a regression between laboratory measurements of dry density and the wet bulk density data collected by the GRAPE (gamma ray attenuation porosity evaluator) at each site.

The product of the linear sedimentation rate (LSR $=\mathrm{cm} / \mathrm{k} . \mathrm{y}$., where k.y. = thousand years) and the dry bulk density (DBD $=\mathrm{g} / \mathrm{cm}^{3}$ ) equals the mass accumulation rate (MAR $=\mathrm{g} / \mathrm{cm}^{2} / \mathrm{k}$.y.). The product of the mass accumulation rate and the relative percentage of each sedimentary component gave the mass accumulation rates for the carbonate (CAR), noncarbonate (NCAR), and eolian (EAR) components of the total flux at each site.

\section{DISCUSSION}

The data collected during this study are presented in a series of tables for each of the guyots drilled during Leg 144. For each guyot, we present the results of the analyses of samples from a specific drillhole in the following order:

First, laboratory determinations of the weight percentage of calcium carbonate and the fine-grained lithogenic (eolian) component of the bulk sediment are given. Accompanying these tables are one or two summary figures for each site. These figures summarize the shipboard measurements of physical properties, shipboard examinations of smear slides (Premoli Silva, Haggerty, Rack, et al., 1993), and the results of laboratory analyses (presented in corresponding tables) for the upper $50 \mathrm{~m}$ in each hole. Next, the two age models developed for each site are given in a common table for each hole. The age models are listed for the individual holes from which they were developed rather than by site, because the "soupy" nature of the sediment recovered by drilling and the changes in core curation procedures that occurred during Leg 144 may have produced major differences between holes.

Finally, the calculations of mass sediment accumulation rates are presented for each guyot (based on the comprehensive and simplified age models) and in corresponding figures that summarize the results of these calculations.

The data and calculations for Limalok Guyot (Hole 871A) are given in Table 1 (laboratory measurements of the weight percentages of calcium carbonate, noncarbonate, and fine-grained lithogenic [eolian] material), Table 2 (two age models used), and Table 3 (sediment accumulation rate calculations using each of the two age models for Hole 871A). Figure 2 illustrates the downhole measurements of bulk physical properties and the comprehensive age model for the upper $50 \mathrm{~m}$ in Hole $871 \mathrm{~A}$. Figure 3 provides a summary of the bulk sediment composition, fossil content, and texture of the upper $50 \mathrm{~m}$ in Hole 871A. Figure 4 illustrates the differences in linear sedimentation and calculated sediment accumulation rates for Hole 871A using the comprehensive and the simplified age models.

The laboratory results from Lo-En Guyot are given in Table 4 (determinations of the weight percentage of calcium carbonate for samples in Holes 872A and 872C), Table 5 (age models for Hole $872 \mathrm{~A}$ ), and Table 6 (accumulation rates calculated for Hole 872A using the comprehensive and simplified age models). Figures 5 and 6 illustrate the downhole measurements of bulk physical properties and the weight percentage of calcium carbonate for the upper $50 \mathrm{~m}$ of Holes $872 \mathrm{~A}$ and $872 \mathrm{C}$, respectively. Figure 7 illustrates the differences in linear sedimentation and calculated sediment accumulation rates for Hole 872A using the comprehensive and the simplified age models. 

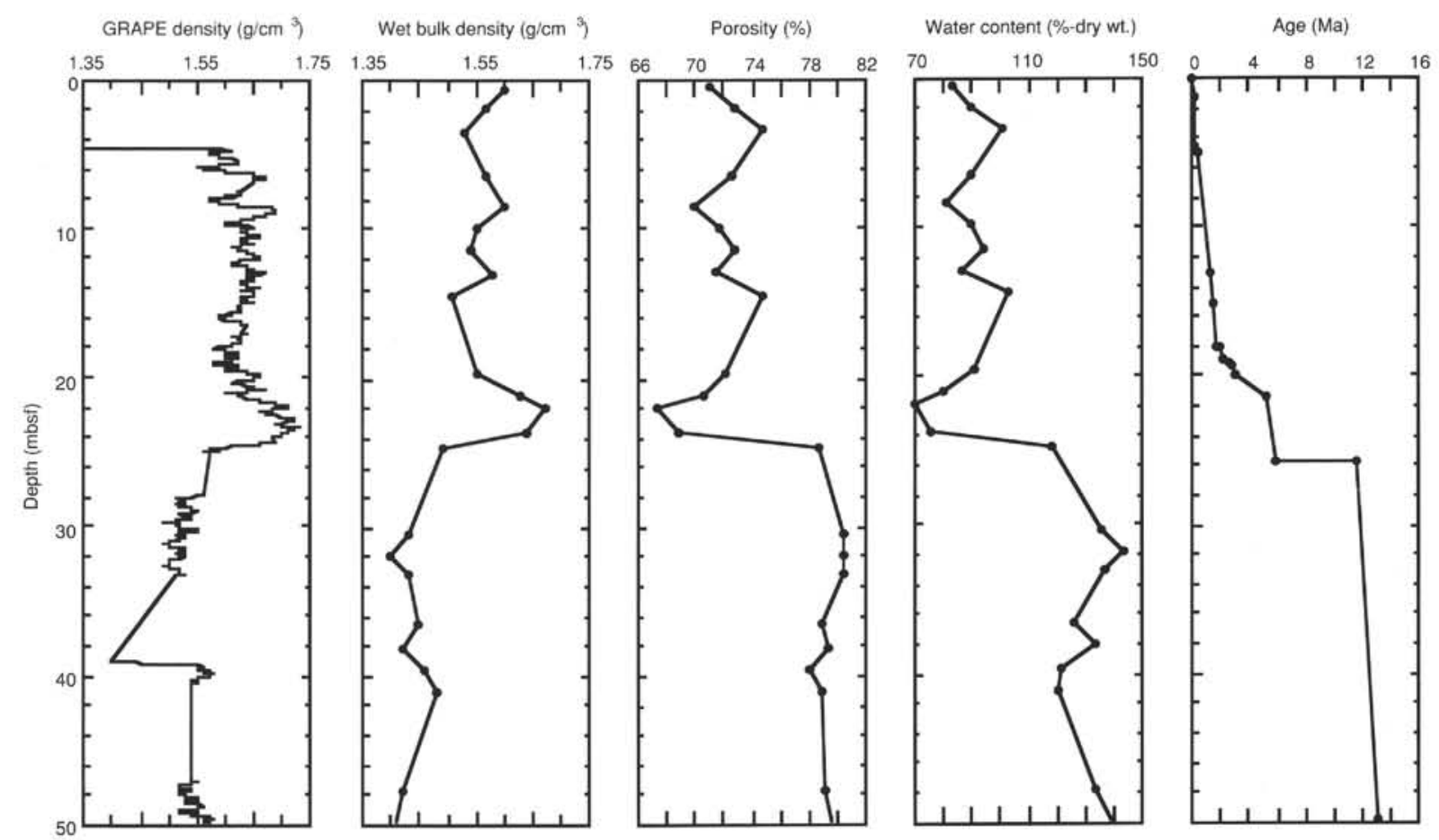

Figure 2. Summary plots of GRAPE density and index properties vs. depth (mbsf), Hole 871A. The comprehensive age model derived from calcareous nannofossil and foraminifer datums (Premoli Silva, Haggerty, Rack, et al., 1993) is also shown.
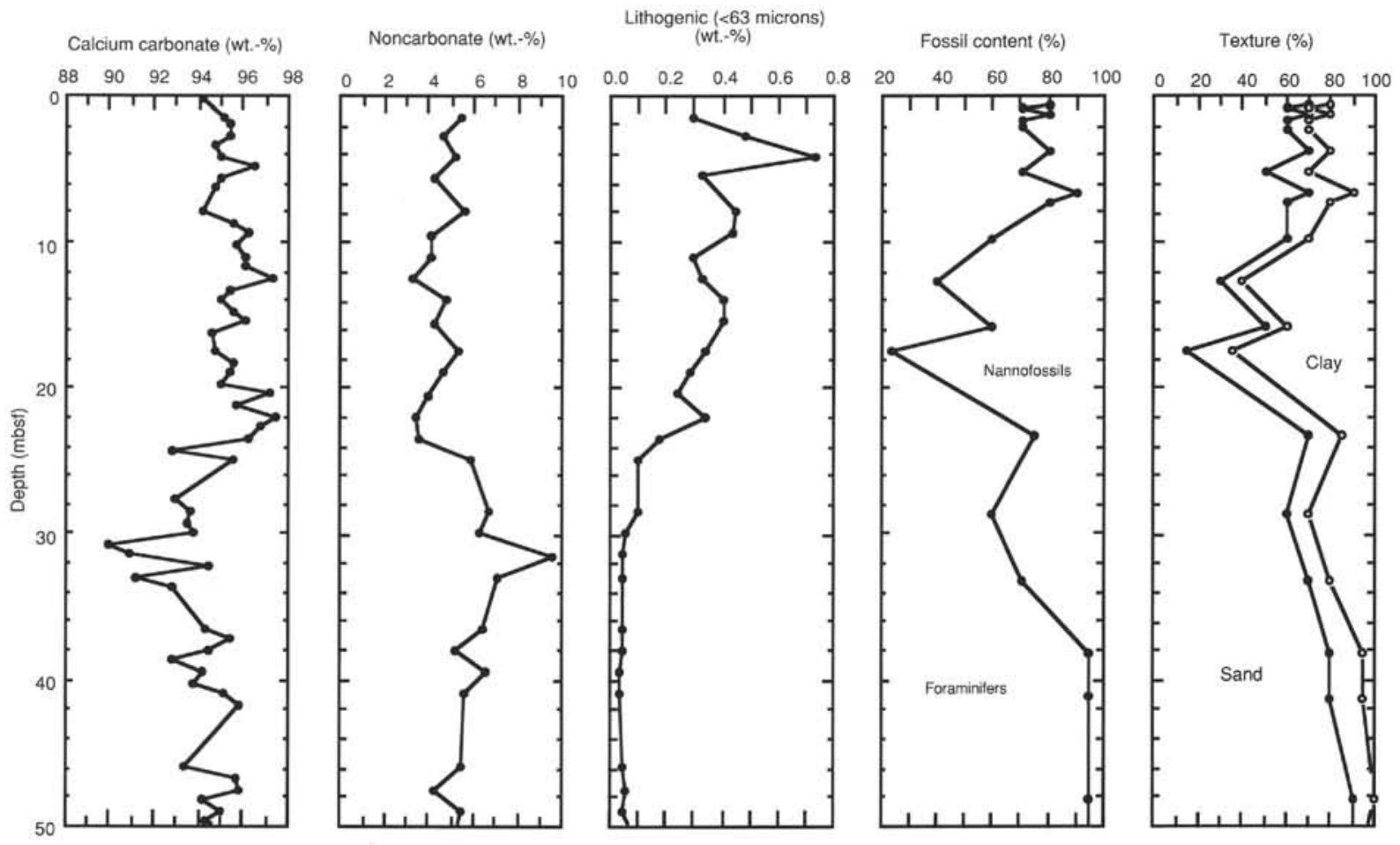

Figure 3. Summary plots of bulk composition, fossil content, and texture (cumulative percentages of sand, silt, and clay) vs. depth (mbsf), Hole 871A. Fossil content and texture are from shipboard smear slide estimates (Premoli Silva, Haggerty, Rack, et al., 1993). 
A

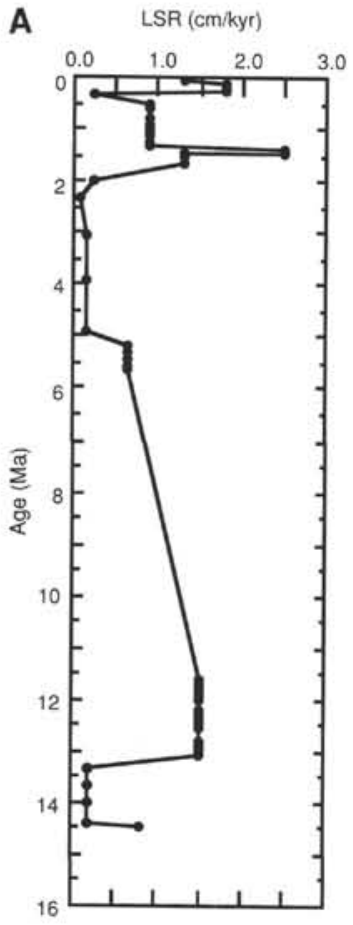

$\operatorname{MAR}\left(\mathrm{g} / \mathrm{cm}^{2} / \mathrm{kyr}\right)$

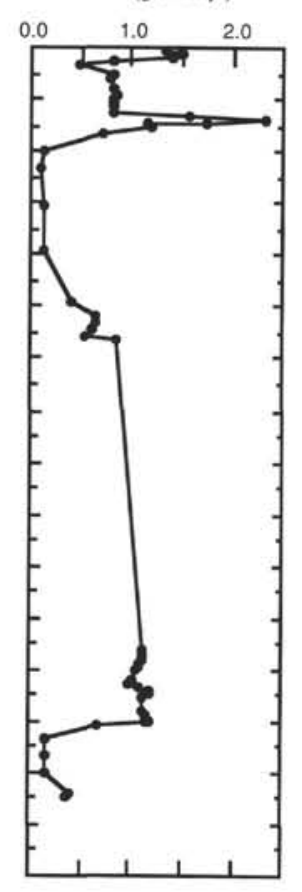

Ave. CAR $\left(g / \mathrm{cm}^{2} / \mathrm{kyr}\right)$

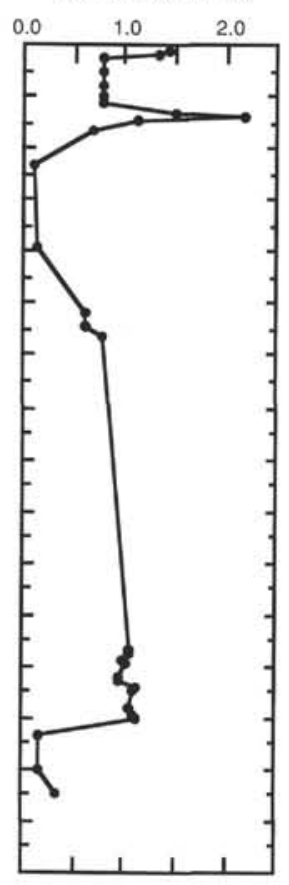

Ave. NCAR $\left(\mathrm{g} / \mathrm{cm}^{2} / \mathrm{kyr}\right)$

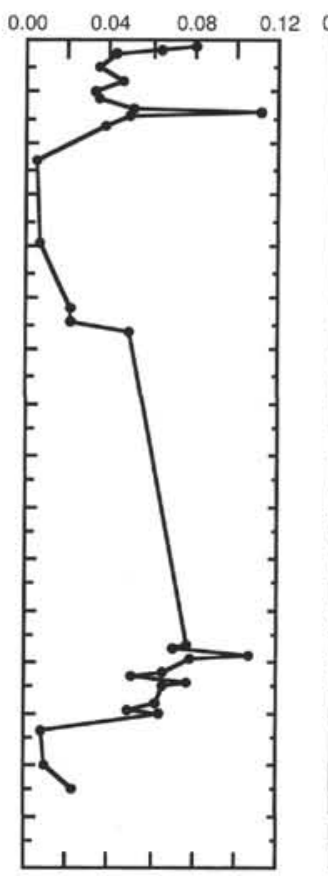

Ave. Eolian MAR $\left(\mathrm{mg} / \mathrm{cm}^{2} / \mathrm{kyr}\right)$

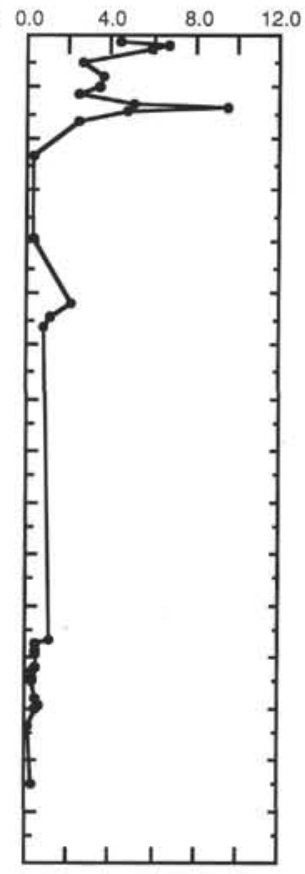

B

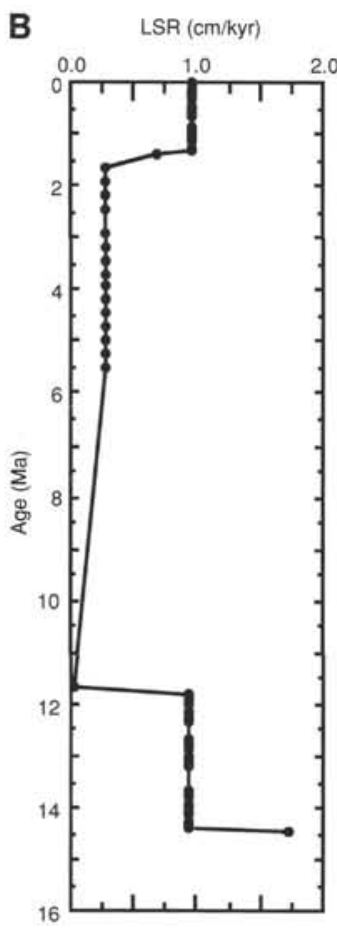

Ave. MAR $\left(\mathrm{g} / \mathrm{cm}^{2} / \mathrm{kyr}\right)$

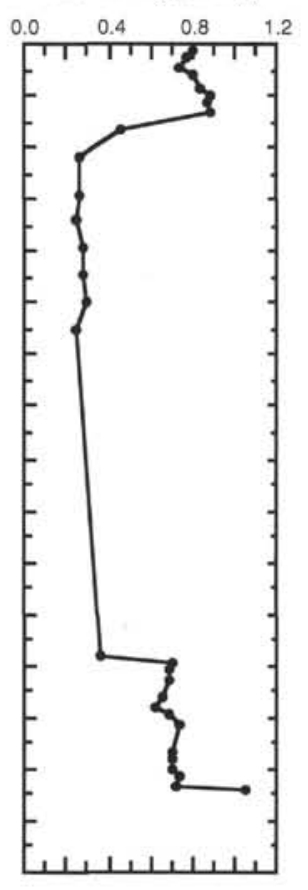

\section{Ave. CAR $\left(g / \mathrm{cm}^{2} / \mathrm{kyr}\right)$}

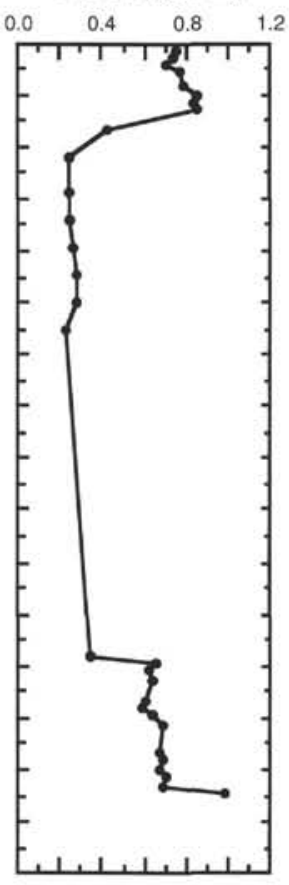

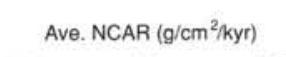

Ave. Eolian MAR (mg/cm ${ }^{2} / \mathrm{kyr}$ )
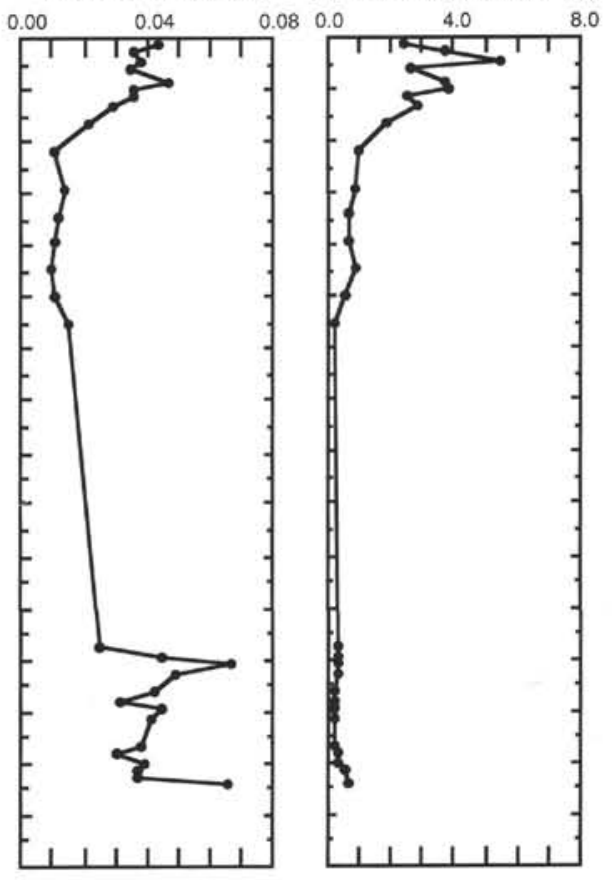

Figure 4. A. Plots of linear sedimentation (LSR), mass (bulk) accumulation (MAR), carbonate accumulation (CAR), noncarbonate accumulation (NCAR), and eolian mass accumulation (Eolian MAR) rates vs. age (Ma), Hole 871A. Calculations are based on the comprehensive age model. Note the change in units for eolian accumulation. B. Plots of linear sedimentation and mass accumulation rates for Hole $871 \mathrm{~A}$, calculated using the simplified age model. Note the change in units for eolian accumulation. 


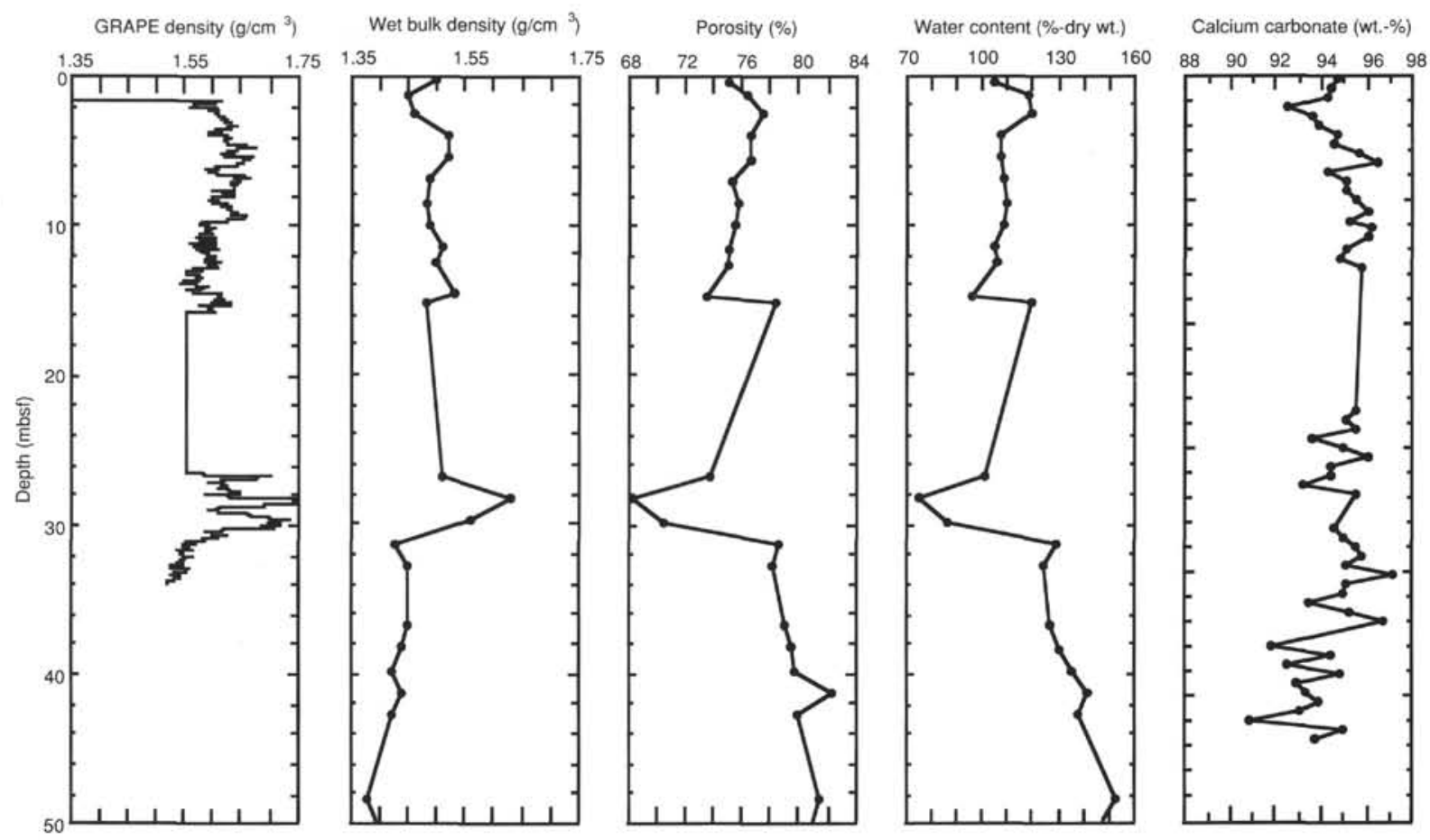

Figure 5. Summary plots of GRAPE density, index properties, and weight percentage of calcium carbonate vs. depth (mbsf), Hole 872A.

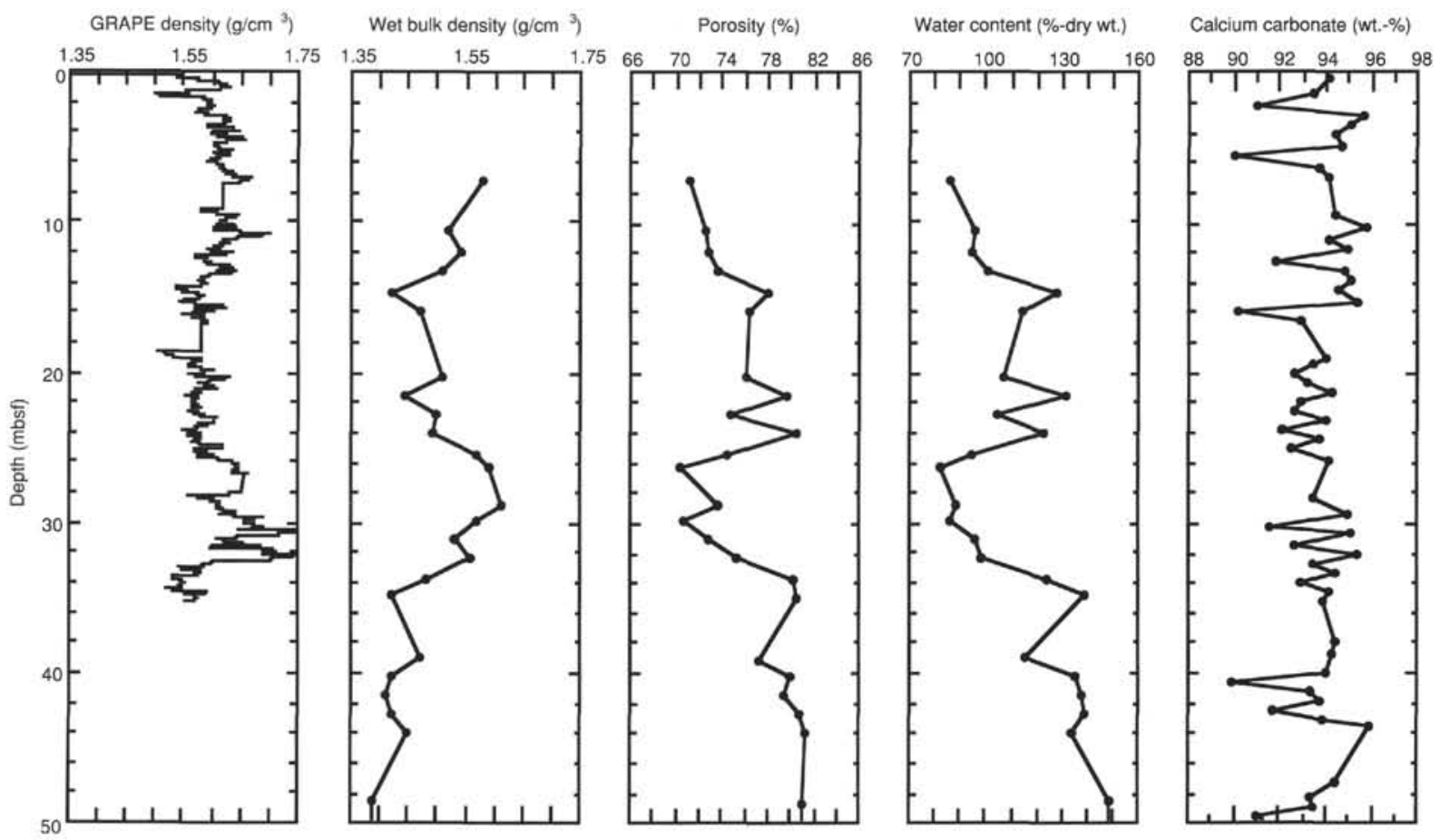

Figure 6. Summary plots of GRAPE density, index properties, and weight percentage of calcium carbonate vs. depth (mbsf), Hole 872C. 
A
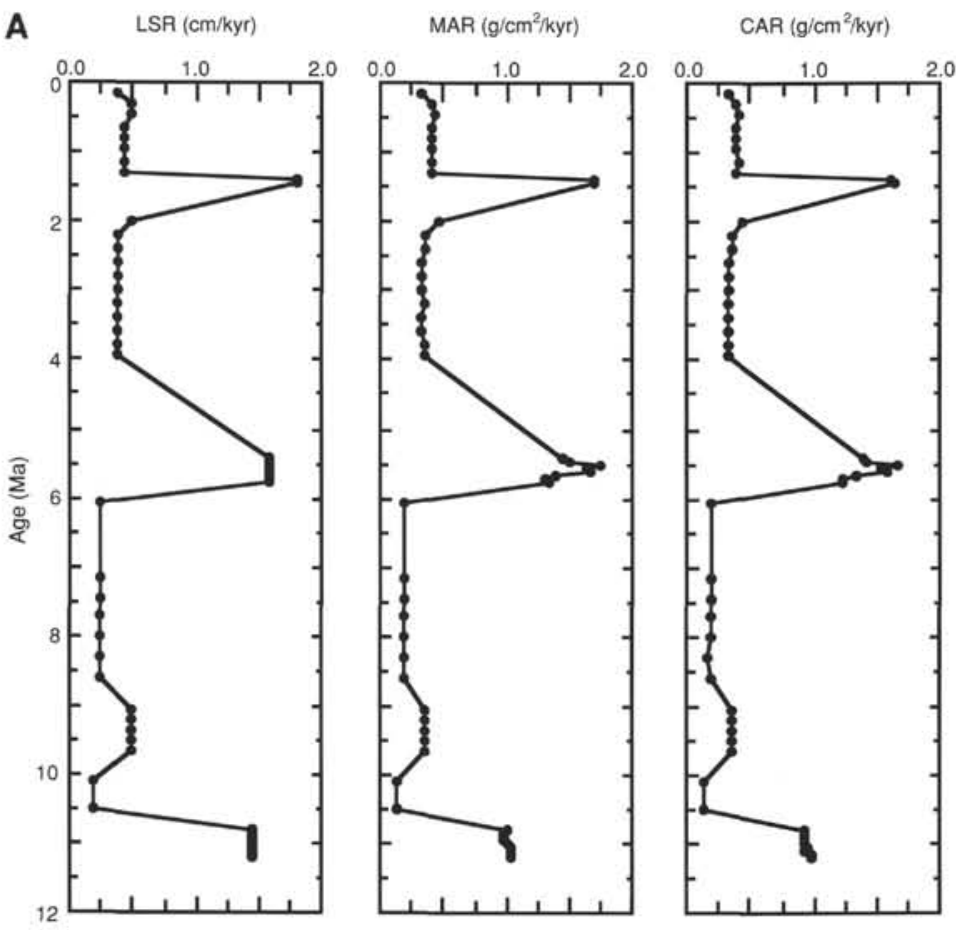

NCAR $\left(g / \mathrm{cm}^{2} / \mathrm{kyr}\right)$

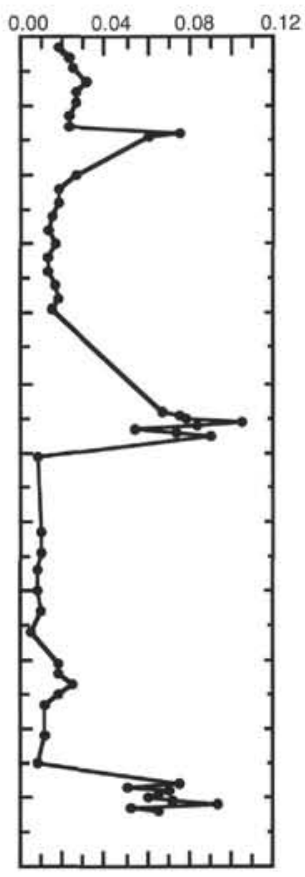

B

$\operatorname{LSR}(\mathrm{cm} / \mathrm{kyr})$
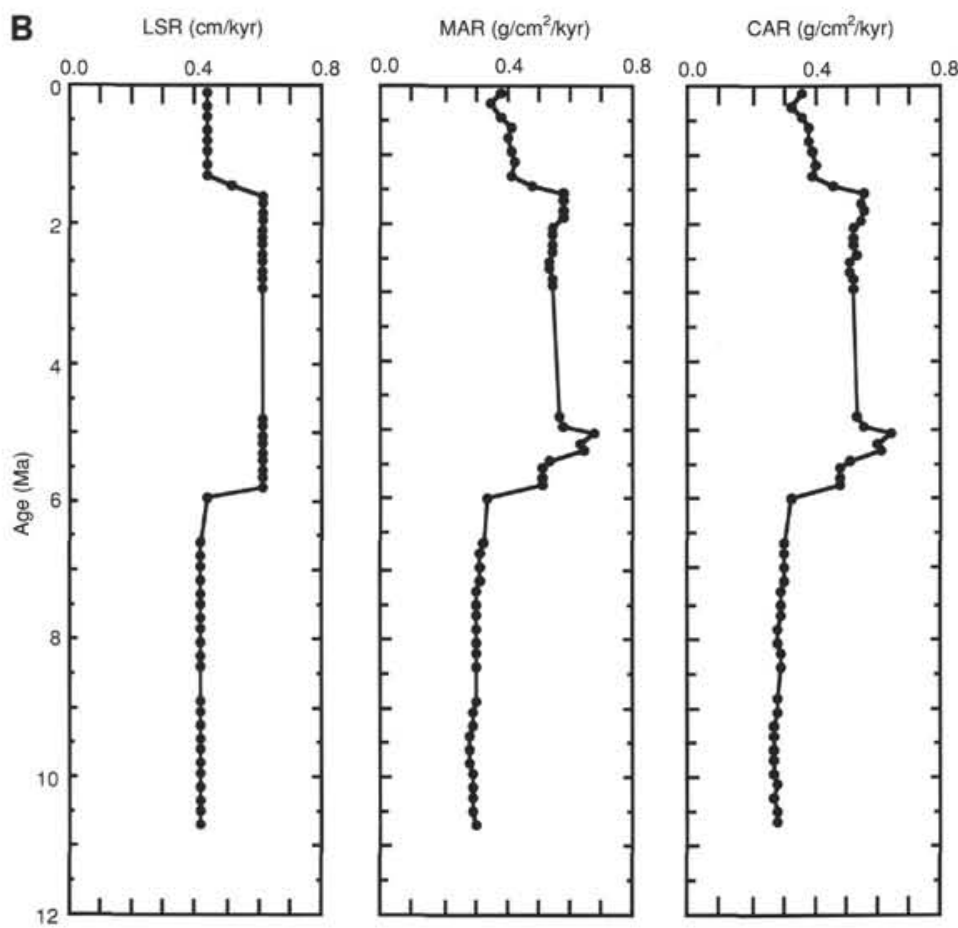

$\operatorname{NCAR}\left(\mathrm{g} / \mathrm{cm}^{2} / \mathrm{kyr}\right)$

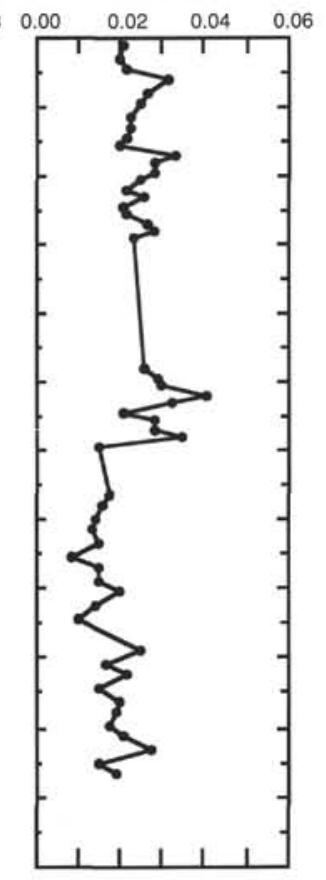

Figure 7. A. Plots of linear sedimentation (LSR), mass (bulk) accumulation (MAR), carbonate accumulation (CAR), and noncarbonate accumulation (NCAR) rates vs. age (Ma), Hole $872 \mathrm{~A}$. Calculations are based on the comprehensive age model. B. Plots of linear sedimentation and mass accumulation rates calculated for Hole $872 \mathrm{~A}$, using the simplified age model. 
Table 1. Calcium carbonate determinations and chemical extractions of fine-grained lithogenic material, Hole 871A.

\begin{tabular}{|c|c|c|c|c|c|c|}
\hline $\begin{array}{l}\text { Core, section, } \\
\text { interval }(\mathrm{cm})\end{array}$ & $\begin{array}{l}\text { Depth } \\
\text { (mbsf) }\end{array}$ & $\begin{array}{l}\mathrm{CaCO}_{3} \\
(\mathrm{wt} \%)\end{array}$ & $\begin{array}{c}\mathrm{CaCO}_{3} \\
\text { (ave.) } \\
(\mathrm{wt} \%)\end{array}$ & $\begin{array}{c}\text { Noncarbonate } \\
\text { (ave.) } \\
\text { (wt\%) }\end{array}$ & $\begin{array}{l}\text { Eolian } \\
\text { (ave.) } \\
\text { (wt\%) }\end{array}$ & $\begin{array}{l}\text { \%Eolian } \\
\text { carb-free }\end{array}$ \\
\hline \multicolumn{7}{|l|}{ 144-871A- } \\
\hline $1 \mathrm{H}-1,24-26$ & 0.24 & 94.19 & & & & \\
\hline $1 \mathrm{H}-\mathrm{I}, 139-14 \mathrm{I}$ & 1.39 & 95.07 & 94.63 & 5.37 & 0.30 & 5.6 \\
\hline $1 \mathrm{H}-2,32-34$ & 1.82 & 95.38 & & & & \\
\hline $1 \mathrm{H}-2,117-119$ & 2.67 & 95.43 & 95.40 & 4.60 & 0.48 & 10.5 \\
\hline $1 \mathrm{H}-3,32-34$ & 3.32 & 94.66 & & & & \\
\hline $1 \mathrm{H}-3,115-117$ & 4.15 & 95.02 & 94.84 & 5.16 & 0.73 & 14.1 \\
\hline $1 \mathrm{H}-4,20-22$ & 4.70 & 96.50 & & & & \\
\hline $1 \mathrm{H}-4,104-106$ & 5.54 & 94.98 & 95.74 & 4.26 & 0.33 & 7.7 \\
\hline IH-5, 22-24 & 6.22 & 94.72 & & & & \\
\hline $2 \mathrm{H}-1,44-46$ & 7.94 & 94.22 & 94.47 & 5.53 & 0.45 & 8.1 \\
\hline $2 \mathrm{H}-1,120-122$ & 8.70 & 95.57 & & & & \\
\hline $2 \mathrm{H} \cdot 2,44-46$ & 9.44 & 96.26 & 95.92 & 4.08 & 0.43 & 10.5 \\
\hline $2 \mathrm{H}-2,120-122$ & 10.20 & 95.71 & & & & \\
\hline $2 \mathrm{H}-3,44-46$ & 10.94 & 96.09 & 95.90 & 4.10 & 0.29 & 7.1 \\
\hline $2 \mathrm{H}-3,120-122$ & 11.70 & 96.06 & & & & \\
\hline $2 \mathrm{H}-4,44-46$ & 12.44 & $\begin{array}{l}97.33 \\
95.38\end{array}$ & 96.69 & 3.31 & 0.32 & 9.7 \\
\hline $\begin{array}{l}2 \mathrm{H}-4,120-122 \\
2 \mathrm{H}-5,44-46\end{array}$ & $\begin{array}{l}13.20 \\
13.94\end{array}$ & $\begin{array}{l}95.38 \\
95.04\end{array}$ & 95.21 & 4.79 & 0.41 & 8.5 \\
\hline $\begin{array}{l}2 \mathrm{H}-5,44-46 \\
2 \mathrm{H}-5,120-122\end{array}$ & $\begin{array}{l}13.94 \\
14.70\end{array}$ & 95.58 & & & & \\
\hline $2 \mathrm{H}-6,44-46$ & 15.44 & 96.09 & 95.83 & 4.17 & 0.41 & 9.8 \\
\hline $2 \mathrm{H}-6,120-122$ & 16.20 & 94.53 & & & & \\
\hline $3 \mathrm{H}-1,46-48$ & 17.46 & 94.75 & 94.64 & 5.36 & 0.34 & 6.3 \\
\hline $3 \mathrm{H}-\mathrm{J}, 12 \mathrm{~J}-123$ & 18.21 & 95.54 & & & & \\
\hline $3 \mathrm{H}-2,45-47$ & 18.95 & 95.34 & 95.44 & 4.56 & 0.29 & 6.3 \\
\hline $3 \mathrm{H}-2,121-123$ & 19.71 & 94.99 & & & & \\
\hline $\begin{array}{l}3 \mathrm{H}-3,45-47 \\
3 \mathrm{H}-3,12 \mathrm{I}-123\end{array}$ & 20.45 & $\begin{array}{l}97.19 \\
95.69\end{array}$ & 96.09 & 3.91 & 0.24 & 6.0 \\
\hline $\begin{array}{l}3 \mathrm{H}-3,12 \mathrm{I}-123 \\
3 \mathrm{H}-4,45-47\end{array}$ & $\begin{array}{l}21.21 \\
21.95\end{array}$ & $\begin{array}{l}95.69 \\
97.48\end{array}$ & 96.59 & 3.41 & 0.34 & 9.9 \\
\hline $\begin{array}{l}3 \mathrm{H}-4,45-47 \\
3 \mathrm{H}-4,121-123\end{array}$ & $\begin{array}{l}21.95 \\
22.71\end{array}$ & $\begin{array}{l}97.48 \\
96.82\end{array}$ & & & & \\
\hline $3 \mathrm{H}-5,45-47$ & 23.45 & 96.18 & 96.50 & 3.50 & 0.18 & 5.1 \\
\hline $3 \mathrm{H}-5,12 \mathrm{I}-123$ & 24.21 & 92.77 & & & & \\
\hline $3 \mathrm{H}-6,45-47$ & 24.95 & 95.59 & 94.18 & 5.82 & 0.10 & 1.7 \\
\hline $4 \mathrm{H}-1,120-122$ & 27.70 & 92.98 & & & & \\
\hline $4 \mathrm{H}-2,45-47$ & 28.45 & 93.62 & 93.30 & 6.70 & 0.09 & 1.4 \\
\hline $4 \mathrm{H}-2,120-122$ & 29.20 & 93.55 & & & & \\
\hline $4 \mathrm{H}-3,45-47$ & 29.95 & 93.82 & 93.68 & 6.32 & 0.05 & 0.8 \\
\hline $4 \mathrm{H}-3,120-122$ & 30.70 & 89.95 & 90.41 & 959 & 0.04 & \\
\hline $\begin{array}{l}4 \mathrm{H}-4,45-47 \\
4 \mathrm{H}-4,120-122\end{array}$ & $\begin{array}{l}31.45 \\
32.20\end{array}$ & $\begin{array}{l}90.87 \\
94.47\end{array}$ & & 9.59 & 0.04 & 0.5 \\
\hline $\begin{array}{l}4 \mathrm{H}-4,120-122 \\
4 \mathrm{H}-5,45-47\end{array}$ & $\begin{array}{l}32.20 \\
32.95\end{array}$ & $\begin{array}{l}94.47 \\
91.24\end{array}$ & 92.85 & 7.15 & 0.05 & \\
\hline $4 \mathrm{H}-5,120-122$ & 33.70 & 92.77 & & & & 0.1 \\
\hline $5 \mathrm{H}-\mathrm{I}, 45-47$ & 36.45 & 94.35 & 93.56 & 6.44 & 0.04 & 0.6 \\
\hline $5 \mathrm{H}-1,120-122$ & 37.20 & 95.33 & & & & \\
\hline $5 \mathrm{H}-2,45-47$ & 37.95 & 94.46 & 94.90 & 5.10 & 0.04 & 0.8 \\
\hline $5 \mathrm{H}-2,120-122$ & 38.70 & 92.80 & & & & \\
\hline $5 \mathrm{H}-3,45-47$ & 39.45 & 94.20 & 93.50 & 6.50 & 0.03 & 0.5 \\
\hline $5 \mathrm{H}-3,120-122$ & 40.20 & 93.75 & & & & \\
\hline $5 \mathrm{H}-4,45-47$ & 40.95 & 95.12 & 94.43 & 5.57 & 0.03 & 0.6 \\
\hline $5 \mathrm{H}-4,120-122$ & 41.70 & 95.77 & & & & \\
\hline $6 \mathrm{H}-1,44-46$ & $\begin{array}{l}45.94 \\
46.70\end{array}$ & $\begin{array}{l}93.32 \\
95.70\end{array}$ & 94.54 & 5.46 & 0.04 & 0.8 \\
\hline $\begin{array}{l}6 \mathrm{H}-1,120-122 \\
6 \mathrm{H}-2,44-46\end{array}$ & $\begin{array}{l}46.70 \\
47.44\end{array}$ & $\begin{array}{l}95.70 \\
95.86\end{array}$ & 95.78 & 4.22 & 0.05 & 1.3 \\
\hline $\begin{array}{l}6 \mathrm{H}-2,44-46 \\
6 \mathrm{H}-2,120-122\end{array}$ & $\begin{array}{l}47.44 \\
48.20\end{array}$ & $\begin{array}{l}95.86 \\
94.18\end{array}$ & & & 0.03 & \\
\hline $6 \mathrm{H}-3,44-46$ & 48.94 & 94.98 & 94.58 & 5.42 & 0.05 & 0.9 \\
\hline $6 \mathrm{H}-3,120-122$ & 49.70 & 94.32 & & & & \\
\hline $6 \mathrm{H}-4,44,46$ & 50.44 & 95.62 & 94.97 & 5.03 & 0.08 & 1.6 \\
\hline $6 \mathrm{H}-4,120-122$ & 51.20 & 94.77 & & & & \\
\hline $6 \mathrm{H}-5,44-46$ & 51.94 & 94.96 & 94.86 & 5.14 & & \\
\hline $\begin{array}{l}6 \mathrm{H}-5,120-122 \\
6 \mathrm{H}-6,44-46\end{array}$ & $\begin{array}{l}52.70 \\
53.44\end{array}$ & $\begin{array}{l}91.03 \\
96.55\end{array}$ & 93.79 & 6.21 & 0.07 & 1.1 \\
\hline
\end{tabular}

Notes: $\mathrm{CaCO}_{3}=$ weight percentage of calcium carbonate in bulk sample; Noncarbonate $=$ percentage of noncarbonate in bulk sample $\left(\right.$ calculated as $100 \%-$ wt. $\%$ CaCO $\left.{ }_{3}\right) ;$ Eolian $=$ weight percentage of the extracted "eolian," or fine-grained $(<63 \mu \mathrm{m})$ lithogenic fraction of the bulk sediment; \%Eolian (carb-free) $=$ weight percentage of the extracted "eolian" fraction relative to the noncarbonate weight percentage, rather than to the total bulk sample. Average values are given where two successive samples were used to conduct the chemical extraction procedure.

The laboratory results from Wodejebato Guyot are given in Table 7 (determinations of the weight percentages of calcium carbonate and finegrained lithogenic [eolian] material for samples in Hole 873B), Table 8 (age models for Hole 873B), and Table 9 (accumulation rates calculated for Hole 873B using the comprehensive and simplified age models).

Figure 8 illustrates the downhole measurements of bulk physical properties and the comprehensive age model for the upper $50 \mathrm{~m}$ in Hole $873 \mathrm{~B}$. Figure 9 provides a summary of the bulk sediment composition, fossil content and texture of the upper $50 \mathrm{~m}$ in Hole $873 \mathrm{~B}$. Figure 10 illustrates the differences in linear sedimentation and calculated sediment accumulation rates for Hole $873 \mathrm{~B}$ using the comprehensive and the simplified age models.

\section{CONCLUSIONS}

The data and figures presented in this report are intended to help in the development of a regional paleoceanographic synthesis for this region of the tropical northwest Pacific Ocean during the Neogene. There are distinct similarities in the downhole profiles of sediment physical properties (GRAPE density, bulk density, porosity, and water content) at each of the three guyots in the Marshall Islands, which surely reflect changes in depositional conditions at intermediate water depths. The timing and ultimate cause of these changes remain uncertain at present, but these issues may be resolved through a synthesis with other data sets. 
Table 2. Age models for Hole 871A.

\begin{tabular}{|c|c|c|c|c|c|}
\hline $\begin{array}{c}\text { Depth } \\
\text { top } \\
\text { (mbsf) }\end{array}$ & $\begin{array}{l}\text { Depth } \\
\text { bottom } \\
\text { (mbsf) }\end{array}$ & $\begin{array}{l}\text { Depth } \\
\text { middle } \\
\text { (mbsf) }\end{array}$ & $\begin{array}{l}\text { Age } \\
(\mathrm{Ma})\end{array}$ & Code & Datum \\
\hline 0.00 & 0.00 & 0.00 & 0.000 & & 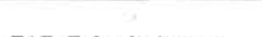 \\
\hline 0.83 & 1.44 & 1.13 & 0.085 & $\mathrm{Cl}$ & FAD E huxleyi acme \\
\hline 4.41 & 4.71 & 4.56 & 0.275 & $\mathrm{C} 2$ & FAD $E$. huxleyi \\
\hline 4.71 & 5.30 & 5.01 & 0.474 & $\mathrm{C} 3$ & LAD P. lacunosa \\
\hline 12.80 & 13.35 & 13.08 & 1.370 & $\mathrm{C} 4$ & LAD $H$. sellii*** \\
\hline 14.80 & 15.35 & 15.08 & 1.450 & C5 & LAD C. macintyrei \\
\hline 17.80 & 18.35 & 18.08 & 1.680 & C6 & FAD G. oceanica \\
\hline 17.80 & 18.35 & 18.08 & 1.900 & $\mathrm{C} 7$ & LAD D. brouweri \\
\hline 18.75 & 19.00 & 18.87 & 2.200 & $\mathrm{C} 8$ & LAD D. asymmetricus \\
\hline 19.00 & 19.30 & 19.15 & 2.600 & C9 & LAD D. tamalis \\
\hline 19.11 & 19.61 & 19.36 & 2.900 & $\mathrm{C} 10$ & LAD D. variabilus \\
\hline 19.10 & 20.60 & 19.85 & 3.100 & $\mathrm{~F} 2$ & FAD G. tosaensis \\
\hline 20.60 & 22.10 & 21.35 & 5.100 & F3 & FAD $S$. dehiscens \\
\hline 25.10 & 26.50 & 25.80 & 5.800 & $\mathrm{~F} 4$ & FAD P. primalis** \\
\hline 25.10 & 26.50 & 25.80 & 11.500 & F5 & LAD $G$. fohsi lobata** \\
\hline 49.10 & 50.60 & 49.85 & 13.100 & F6 & FAD G. fohsi lobata \\
\hline 52.00 & 53.50 & 52.75 & 14.400 & C11 & LAD $S$, heteromorphus** \\
\hline
\end{tabular}

Notes: Age models were generated using the midpoint depth (mbsf) for each datum. Double asterisks $\left(^{* *}\right)$ indicate the datums that were used to generate the "simplified" version of the age model. $\mathrm{FAD}=$ first appearance datum, and $\mathrm{LAD}=$ last appearance datum. Codes are taken from Premoli Silva, Haggerty, Rack, et al. (1993). $\mathrm{C}=$ nannofossil datum, and $\mathrm{F}=$ foraminifer datum.

\section{ACKNOWLEDGMENTS}

This research was supported by a grant from JOI-USSAC and a Canadian JGOFS post-doctoral fellowship to the first author. We thank Isabella Premoli Silva, Janet Haggerty, and the Leg 144 Scientific Party for discussions and cooperation related to this study. We also thank Monty Lawer and Tanya Shank of the Department of Geology and Geophysics, Texas A\&M University, for their assistance with data collection. Finally, we thank the Ocean Drilling Program shipboard technicians and staff for their help and encouragement during Leg 144.

\section{REFERENCES $*$}

Arimoto, R., Duce, R.A., Ray, B.J., and Unni, C.K., 1985. Atmospheric trace elements at Enewetak Atoll: 2 . Transport to the ocean by wet and dry deposition. J. Geophys. Res., 90:2391-2408.
Clemens, S.C., and Prell, W.L., 1990. Late Pleistocene variability of Arabian Sea summer monsoon winds and continental aridity: eolian records from the lithogenic component of deep-sea sediments. Paleoceanography, 5:109-145.

Duce, R.A., Arimoto, R., Ray, B.J., Unni, C.K., and Harder, P.J., 1983. Atmospheric trace elements at Enewetak Atoll: 1 . Concentrations, sources, and temporal variability. J. Geophys. Res., 88:5321-5342.

Duce, R.A., Unni, C.K., Ray, B.J., Prospero, J.M., and Merrill, J.T., 1980. Long-range atmospheric transport of soil dust from asia to the Tropical North Pacific: temporal variability. Science, 209:1522-1524.

Janecek, T.R., and Rea, D.K., 1983. Eolian deposition in the northeast Pacific Ocean: Cenozoic history of atmospheric circulation. Geol. Soc. Am. Bull., 94:730-738.

Lincoln, J.M., Pringle, M.S., and Premoli-Silva, I., 1993. Early and Late Cretaceous volcanism and reef-building in the Marshall Islands. In Pringle, M.S., Sager, W.W., Sliter, W.V., and Stein, S. (Eds.), The Mesozoic Pacific: Geology, Tectonics, and Volcanism. Geophys. Monogr., Am. Geophys. Union, 77:279-305.

Premoli Silva, I., Haggerty, J., Rack, F., et al., 1993. Proc. ODP, Init. Repts., 144: College Station, TX (Ocean Drilling Program).

Rea, D.K., and Janecek, T.R., 1981. Mass-accumulation rates of the nonauthigenic inorganic crystalline (eolian) component of deep-sea sediments from the western mid-Pacific Mountains, Deep Sea Drilling Project Site 463. In Theide, J., Vallier, T.L., et al., Init. Repts. DSDP, 62: Washington (U.S. Govt. Printing Office), 653-659.

Uematsu, M., Duce, R.A., Prospero, J.M., Chen, L., Merrill, J.T., and McDonald, R.L., 1983. Transport of mineral aerosol from Asia over the North Pacific Ocean. J. Geophys. Res., 88:5343-5352.

van Waasbergen, R.J., and Winterer, E.L., 1993. Summit geomorphology of Western Pacific guyots. In Pringle, M.S., Sager, W.W., Sliter, W.V., and Stein, S. (Eds.), The Mesozoic Pacific: Geology, Tectonics, and Volcanism. Geophys. Monogr., Am. Geophys. Union, 77:335-366.

\footnotetext{
-Abbreviations for names of organizations and publications in ODP reference lists follow the style given in Chemical Abstracts Service Source Index (published by American Chemical Society).
}

\author{
Date of initial receipt: 1 February 1994 \\ Date of acceptance: 15 September 1994 \\ Ms 144SR-059



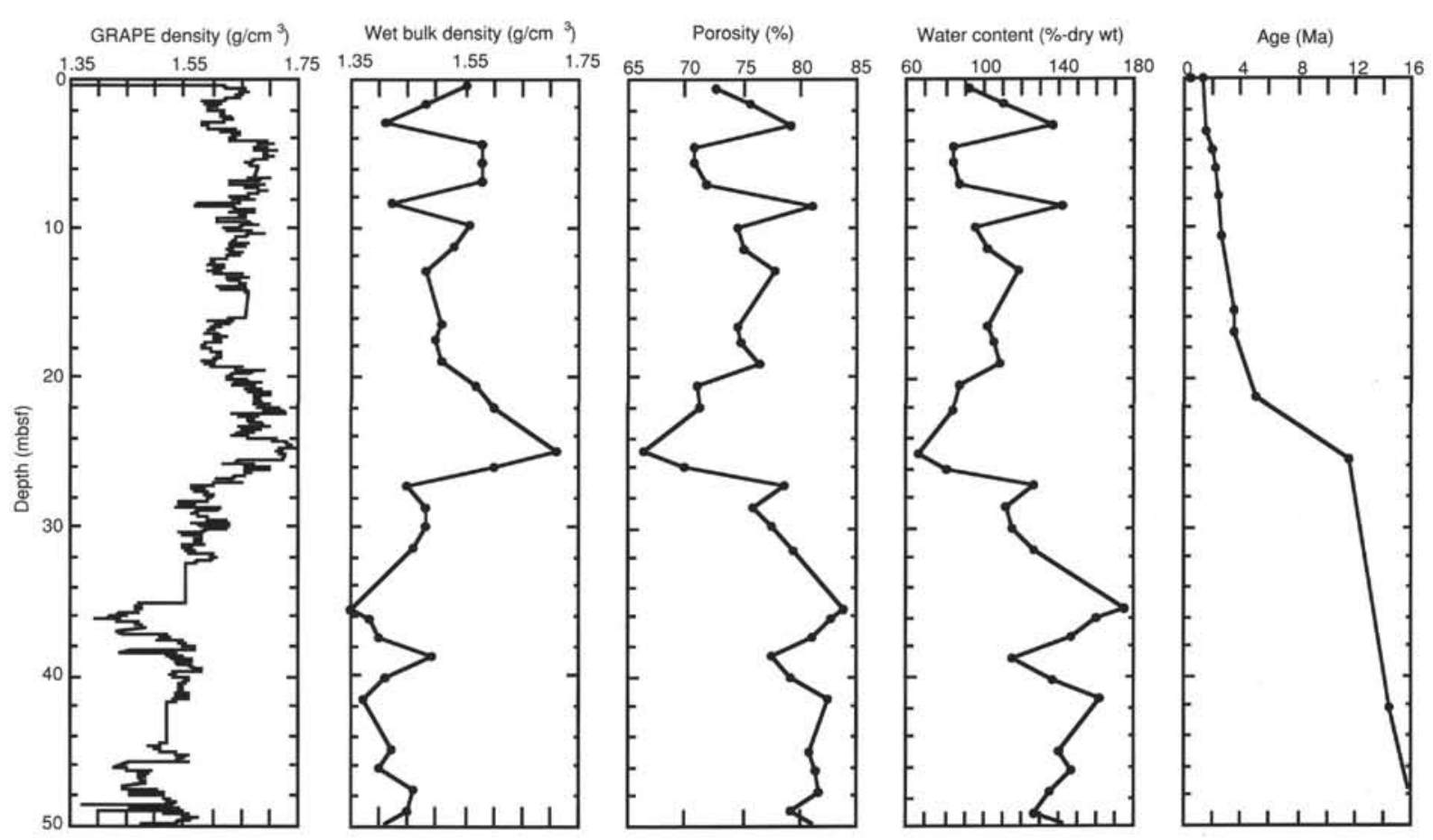

Figure 8. Summary plots of GRAPE density and index properties vs. depth (mbsf), Hole 873B. The comprehensive age model derived from calcareous nannofossil and foraminifer datums (Premoli Silva, Haggerty, Rack, et al., 1993) is also shown.
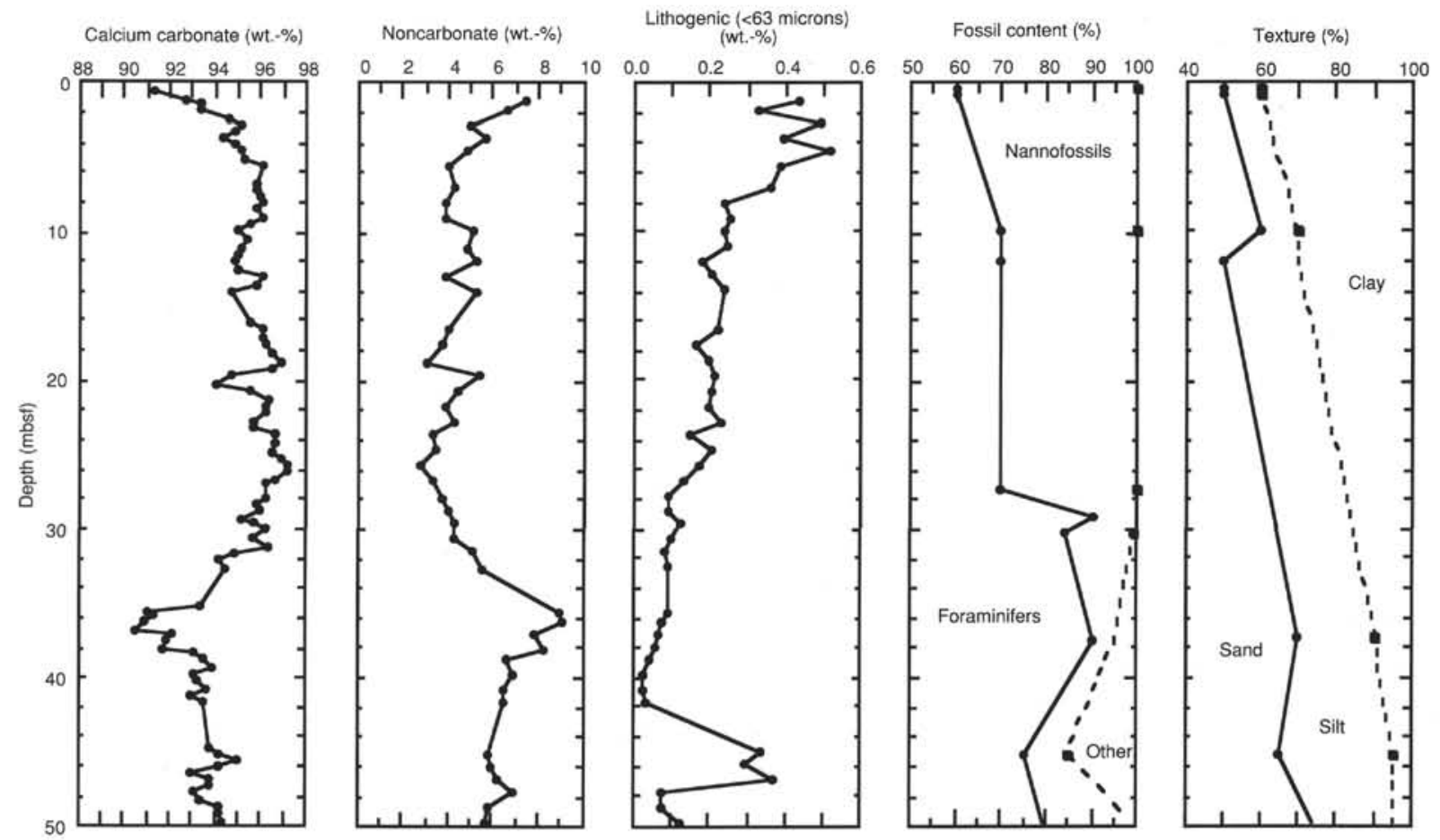

Figure 9. Summary plots of bulk composition, fossil content, and texture vs. depth, Hole 873B. Fossil content and texture are from shipboard smear-slide estimates (Premoli Silva, Haggerty, Rack, et al., 1993). 
A

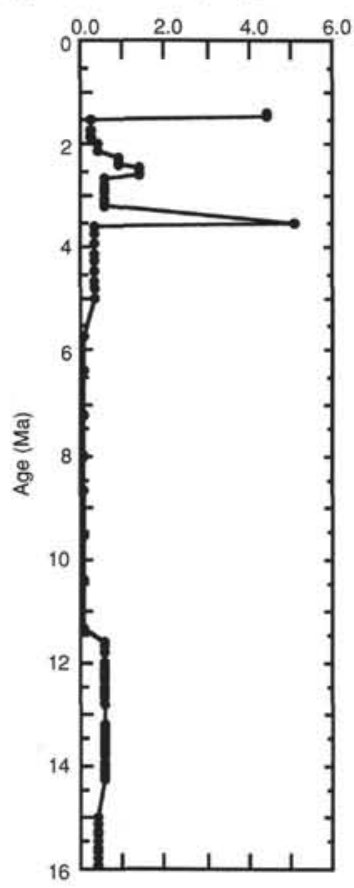

MAR $\left(\mathrm{g} / \mathrm{cm}^{2} / \mathrm{kyr}\right)$

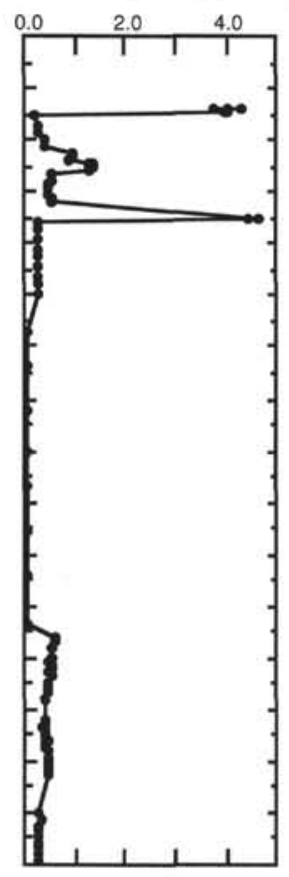

Ave. CAR $\left(g / \mathrm{cm}^{2} / \mathrm{kyr}\right)$

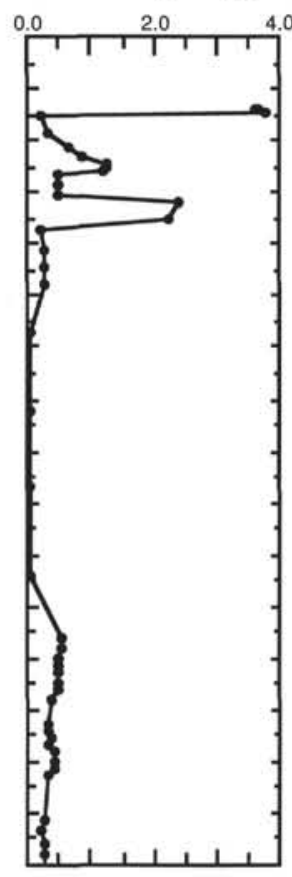

Ave. NCAR $\left(\mathrm{g} / \mathrm{cm}^{2} / \mathrm{kyr}\right)$

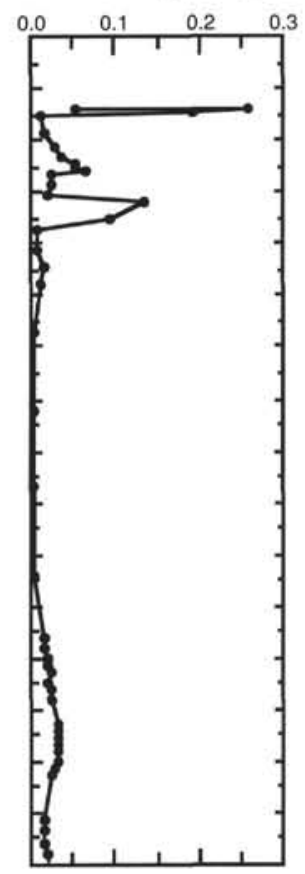

Ave. Eolian MAR ( $\mathrm{mg} / \mathrm{cm}^{2} / \mathrm{kyr}$ )

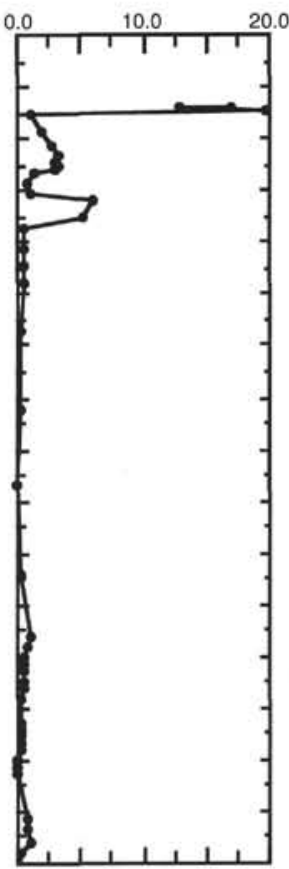

B

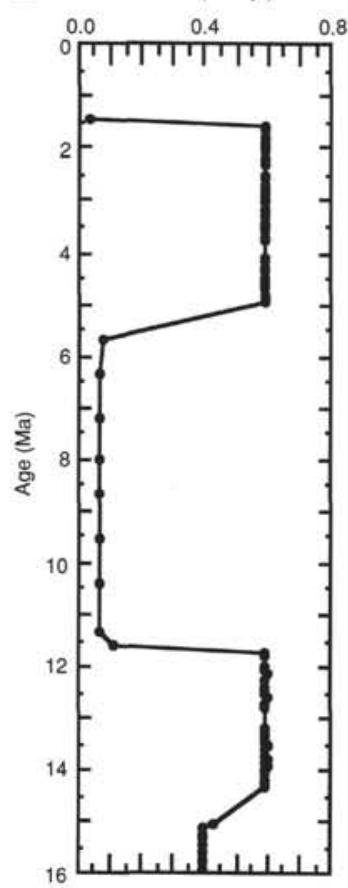

Ave. MAR $\left(\mathrm{g} / \mathrm{cm}^{2} / \mathrm{kyr}\right.$ )

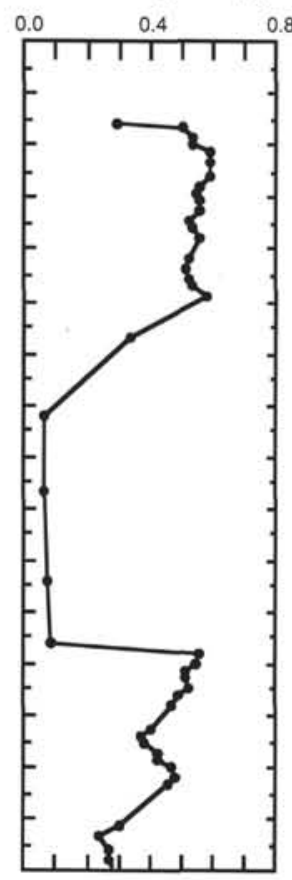

Ave. CAR $\left(\mathrm{g} / \mathrm{cm}^{2} / \mathrm{kyr}\right)$

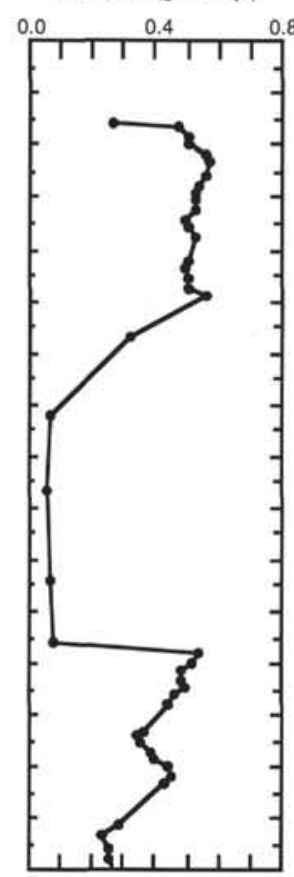

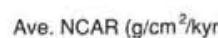

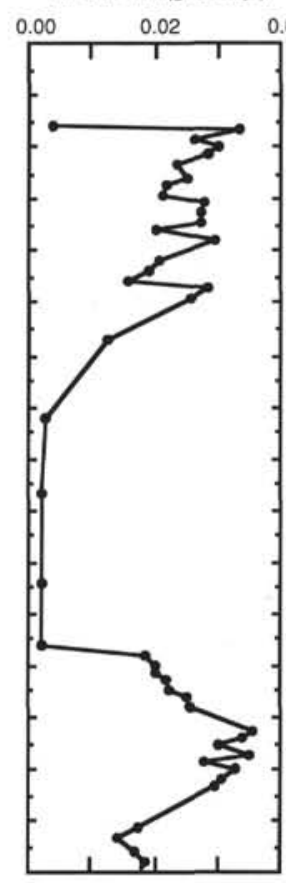

Ave. Eolian MAR $\left(\mathrm{mg} / \mathrm{cm}^{2} / \mathrm{kyr}\right)$

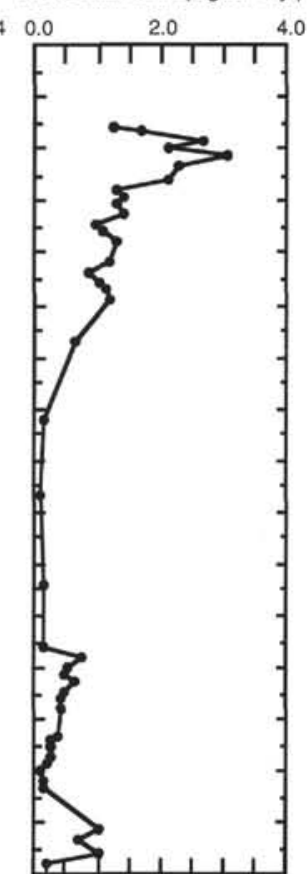

Figure 10. A. Plots of linear sedimentation (LSR), mass (bulk) accumulation (MAR), carbonate accumulation (CAR), noncarbonate accumulation (NCAR), and eolian mass accumulation (Eolian MAR) rates vs. age (Ma), Hole 873B. Calculations are based on the comprehensive age model. Note the change in units for eolian accumulation. B. Plots of linear sedimentation and mass accumulation rates for Hole 873B, calculated using the simplified age model. Note the change in units for eolian accumulation. 
Table 3. Linear sedimentation and mass accumulation rates, Hole 871A, calculated using the comprehensive and simplified age models.

\begin{tabular}{|c|c|c|c|c|c|c|c|c|c|c|}
\hline $\begin{array}{l}\text { Core, section } \\
\text { interval }(\mathrm{cm})\end{array}$ & $\begin{array}{l}\text { Depth } \\
\text { (mbsf) }\end{array}$ & $\begin{array}{l}\text { Age } \\
\text { (Ma) }\end{array}$ & $\begin{array}{c}\text { LSR } \\
\text { (cm/k.y.) }\end{array}$ & $\begin{array}{c}\text { DBD } \\
\left(\mathrm{g} / \mathrm{cm}^{3}\right)\end{array}$ & MAR & $\begin{array}{l}\text { MAR } \\
\text { (ave.) }\end{array}$ & CAR & $\begin{array}{l}\text { CAR } \\
\text { (ave.) }\end{array}$ & $\begin{array}{l}\text { NCAR } \\
\text { (ave.) }\end{array}$ & $\begin{array}{r}\text { EAR } \\
\text { (ave.) }\end{array}$ \\
\hline \multirow{2}{*}{\multicolumn{11}{|c|}{$\begin{array}{l}\text { Comprehensive model: } \\
\text { 144-871A- }\end{array}$}} \\
\hline & 0.24 & 0.018 & 1.330 & 0.85 & 1.131 & & 1.065 & & & \\
\hline $\begin{array}{l}1 \mathrm{H}-1,24 \\
1 \mathrm{H}-1,139\end{array}$ & 1.39 & 0.099 & 1.805 & 0.83 & 1.498 & 1.314 & 1.424 & 1.244 & 0.071 & 0.0039 \\
\hline $1 \mathrm{H}-2.32$ & 1.82 & 0.123 & 1.805 & 0.83 & 1.498 & & 1.429 & & & \\
\hline $1 \mathrm{H}-2,117$ & 2.67 & 0.170 & 1.805 & 0.80 & 1.444 & 1.471 & 1.378 & 1.403 & 0.068 & 0.0071 \\
\hline $1 \mathrm{H}-3,32$ & 3.32 & 0.206 & 1.805 & 0.76 & 1.372 & & 1.299 & & & \\
\hline $1 \mathrm{H}-3,115$ & 4.15 & 0.252 & 1.805 & 0.80 & 1.444 & 1.408 & 1.372 & 1.335 & 0.073 & 0.0103 \\
\hline $\mathrm{IH}-4,20$ & 4.70 & 0.337 & 0.226 & 0.84 & 0.190 & & 0.183 & & & \\
\hline $\mathrm{IH}-4,104$ & 5.54 & 0.533 & 0.901 & 0.86 & 0.775 & 0.482 & 0.736 & 0.426 & 0.021 & 0.0016 \\
\hline IH $-5,22$ & 6.22 & 0.608 & 0.901 & 0.92 & 0.829 & & 0.785 & & & \\
\hline $2 \mathrm{H}-1,44$ & 7.94 & 0.799 & 0.901 & 0.83 & 0.747 & 0.788 & 0.704 & 0.744 & 0.044 & 0.0035 \\
\hline $2 \mathrm{H}-1,120$ & 8.70 & 0.884 & 0.901 & 0.99 & 0.892 & & 0.852 & & & \\
\hline $2 \mathrm{H}-2,44$ & 9.44 & 0.966 & 0.901 & 0.87 & 0.784 & 0.838 & 0.755 & 0.804 & 0.034 & 0.0036 \\
\hline $2 \mathrm{H}-2,120$ & 10.20 & 1.050 & 0.901 & 0.93 & 0.838 & & 0.802 & & & \\
\hline $2 \mathrm{H}-3,44$ & 10.94 & 1.132 & 0.901 & 0.91 & 0.820 & 0.829 & 0.788 & 0.795 & 0.034 & 0.0024 \\
\hline $2 \mathrm{H}-3,120$ & 11.70 & 1.217 & 0.901 & 0.94 & 0.847 & & 0.814 & & & \\
\hline $2 \mathrm{H}-4,44$ & 12.44 & 1.299 & 0.901 & 0.91 & 0.820 & 0.834 & 0.798 & 0.806 & 0.028 & 0.0027 \\
\hline $2 \mathrm{H}-4,120$ & 13.20 & 1.375 & 2.500 & 0.92 & 2.300 & & 2.194 & & & \\
\hline $2 \mathrm{H}-5,44$ & 13.94 & 1.404 & 2.500 & 0.93 & 2.325 & 2.312 & 2.210 & 2.202 & 0.111 & 0.0094 \\
\hline $2 \mathrm{H}-5,120$ & 14.70 & 1.435 & 2.500 & 0.92 & 2.300 & & 2.198 & & & \\
\hline $2 \mathrm{H}-6,44$ & 15.44 & 1.478 & 1.304 & 0.89 & 1.161 & 1.730 & 1.115 & 1.658 & 0.072 & 0.0070 \\
\hline $2 \mathrm{H}-6,120$ & 16.20 & 1.536 & 1.304 & 0.91 & 1.186 & & 1.121 & & & \\
\hline $3 \mathrm{H}-1,46$ & 17.46 & 1.633 & 1.304 & 0.90 & 1.174 & 1.180 & 1.112 & 1.117 & 0.063 & 0.0040 \\
\hline $3 \mathrm{H}-\mathrm{I}, 12 \mathrm{I}$ & 18.21 & 1.949 & 0.263 & 0.88 & 0.231 & & 0.221 & & & \\
\hline $3 \mathrm{H}-2,45$ & 18.95 & 2.314 & 0.070 & 0.85 & 0.060 & 0.145 & 0.057 & 0.139 & 0.007 & 0.0004 \\
\hline $3 \mathrm{H}-2,12 \mathrm{I}$ & 19.71 & 3.043 & 0.158 & 0.95 & 0.150 & & 0.143 & & & \\
\hline $3 \mathrm{H}-3,45$ & 20.45 & 3.900 & 0.158 & 0.92 & 0.145 & 0.148 & 0.141 & 0.142 & 0.006 & 0.0003 \\
\hline $3 \mathrm{H}-3,12 \mathrm{I}$ & 21.21 & 4.913 & 0.158 & 0.94 & 0.149 & & 0.142 & & & \\
\hline $3 \mathrm{H}-4,45$ & 21.95 & 5.194 & 0.636 & 1.00 & 0.636 & 0.392 & 0.620 & 0.379 & 0.013 & 0.0013 \\
\hline $3 \mathrm{H}-4,12 \mathrm{I}$ & 22.71 & 5.314 & 0.636 & 1.01 & 0.642 & & 0.622 & & & \\
\hline $3 \mathrm{H}-5,45$ & 23.45 & 5.430 & 0.636 & 1.02 & 0.648 & 0.645 & 0.624 & 0.623 & 0.023 & 0.0011 \\
\hline $3 \mathrm{H}-5,12 \mathrm{I}$ & 24.21 & 5.550 & 0.636 & 0.93 & 0.591 & & 0.549 & & & \\
\hline $3 \mathrm{H}-6,45$ & 24.95 & 5.666 & 0.636 & 0.81 & 0.515 & 0.553 & 0.492 & 0.521 & 0.032 & 0.0006 \\
\hline $4 \mathrm{H}-1,120$ & 27.70 & 11.626 & 1.503 & 0.77 & 1.157 & & 1.076 & & & \\
\hline $4 \mathrm{H}-2,45$ & 28.45 & 11.676 & 1.503 & 0.75 & 1.127 & 1.142 & 1.055 & 1.066 & 0.077 & 0.0011 \\
\hline $4 \mathrm{H}-2,120$ & 29.20 & 11.726 & 1.503 & 0.77 & 1.157 & & 1.083 & & & \\
\hline $4 \mathrm{H}-3,45$ & 29.95 & 11.776 & 1.503 & 0.75 & 1.127 & 1.142 & 1.058 & 1.070 & 0.072 & 0.0006 \\
\hline $4 \mathrm{H}-3,120$ & 30.70 & 11.826 & 1.503 & 0.74 & 1.112 & & 1.000 & & & \\
\hline $4 \mathrm{H}-4,45$ & 31.45 & 11.876 & 1.503 & 0.75 & 1.127 & 1.120 & 1.024 & 1.012 & 0.107 & 0.0005 \\
\hline $4 \mathrm{H}-4,120$ & 32.20 & 11.926 & 1.503 & 0.72 & 1.082 & & 1.022 & & & \\
\hline $4 \mathrm{H}-5,45$ & 32.95 & 11.976 & 1.503 & 0.75 & 1.127 & 1.105 & 1.029 & 1.026 & 0.079 & 0.0005 \\
\hline $4 \mathrm{H}-5,120$ & 33.70 & 12.026 & 1.503 & 0.71 & 1.067 & & 0.990 & & & \\
\hline $5 \mathrm{H}-1,45$ & 36.45 & 12.208 & 1.504 & 0.69 & 1.037 & 1.052 & 0.978 & 0.984 & 0.068 & 0.0004 \\
\hline $5 \mathrm{H}-1,120$ & 37.20 & 12.258 & 1.503 & 0.68 & 1.022 & & 0.974 & & & \\
\hline $5 \mathrm{H}-2,45$ & 37.95 & 12.308 & 1.503 & 0.65 & 0.977 & 0.999 & 0.923 & 0.948 & 0.051 & 0.0004 \\
\hline $5 \mathrm{H}-2,120$ & 38.70 & 12.358 & 1.503 & 0.67 & 1.007 & & 0.935 & & & \\
\hline $5 \mathrm{H}-3,45$ & 39.45 & 12.408 & 1.503 & 0.80 & 1.202 & 1.105 & 1.133 & 1.033 & 0.072 & 0.0004 \\
\hline $5 \mathrm{H}-3,120$ & 40.20 & 12.458 & 1.503 & 0.79 & 1.187 & & 1.113 & & & \\
\hline $5 \mathrm{H}-4,45$ & 40.95 & 12.508 & 1.503 & 0.79 & 1.187 & 1.187 & 1.129 & 1.121 & 0.066 & 0.0004 \\
\hline $5 \mathrm{H}-4,120$ & 41.70 & 12.558 & 1.503 & 0.77 & 1.157 & & 1.108 & & & \\
\hline $6 \mathrm{H}-1,44$ & 45.94 & 12.840 & 1.503 & 0.74 & 1.112 & 1.135 & 1.038 & 1.073 & 0.062 & 0.0005 \\
\hline $6 \mathrm{H}-1,120$ & 46.70 & 12.890 & 1.503 & 0.77 & 1.159 & & 1.109 & & & \\
\hline $6 \mathrm{H}-2,44$ & 47.44 & 12.940 & 1.503 & 0.76 & 1.142 & 1.151 & 1.095 & 1.102 & 0.049 & 0.0006 \\
\hline $6 \mathrm{H}-2,120$ & 48.20 & 12.990 & 1.503 & 0.77 & 1.157 & & 1.090 & & & \\
\hline $6 \mathrm{H}-3,44$ & 48.94 & 13.040 & 1.503 & 0.76 & 1.142 & 1.150 & 1.085 & 1.087 & 0.062 & 0.0006 \\
\hline $6 \mathrm{H}-3,120$ & 49.70 & 13.090 & 1.503 & 0.81 & 1.217 & & 1.148 & & & \\
\hline $6 \mathrm{H}-4,44$ & 50.44 & 13.365 & 0.223 & 0.78 & 0.174 & 0.696 & 0.166 & 0.661 & 0.035 & 0.0006 \\
\hline $6 \mathrm{H}-4,120$ & 51.20 & 13.705 & 0.223 & 0.77 & 0.172 & & 0.163 & & & \\
\hline $6 \mathrm{H}-5,44$ & 51.94 & 14.037 & 0.223 & 0.78 & 0.174 & 0.173 & 0.165 & 0.164 & 0.009 & \\
\hline $6 \mathrm{H}-5,120$ & 52.70 & 14.378 & 0.223 & 0.80 & 0.178 & & 0.162 & & & \\
\hline $6 \mathrm{H}-6,44$ & 53.44 & 14.485 & 0.810 & 0.79 & 0.640 & 0.409 & 0.618 & 0.384 & 0.025 & 0.0003 \\
\hline $\begin{array}{l}\text { Simplified mode } \\
144-871 \mathrm{~A}-\end{array}$ & & & & & & & & & & \\
\hline $1 \mathrm{H}-1,24$ & 0.24 & 0.025 & 0.956 & 0.85 & 0.813 & & 0.766 & & & \\
\hline IH-I, 139 & 1.39 & 0.146 & 0.954 & 0.83 & 0.792 & 0.802 & 0.753 & 0.759 & 0.043 & 0.0024 \\
\hline $1 \mathrm{H}-2,32$ & 1.82 & 0.191 & 0.956 & 0.83 & 0.793 & & 0.756 & & & \\
\hline $1 \mathrm{H}-2,117$ & 2.67 & 0.280 & 0.954 & 0.80 & 0.763 & 0.778 & 0.728 & 0.742 & 0.036 & 0.0037 \\
\hline $1 \mathrm{H}-3,32$ & 3.32 & 0.348 & 0.956 & 0.76 & 0.726 & & 0.688 & & & \\
\hline $1 \mathrm{H}-3,115$ & 4.15 & 0.435 & 0.954 & 0.80 & 0.763 & 0.745 & 0.725 & 0.706 & 0.038 & 0.0054 \\
\hline $1 \mathrm{H}-4,20$ & 4.70 & 0.492 & 0.955 & 0.84 & 0.802 & & 0.774 & & & \\
\hline $1 \mathrm{H}-4,104$ & 5.54 & 0.580 & 0.955 & 0.86 & 0.821 & 0.811 & 0.780 & 0.777 & 0.035 & 0.0027 \\
\hline $1 \mathrm{H}-5,22$ & 6.22 & 0.651 & 0.955 & 0.92 & 0.879 & & 0.832 & & & \\
\hline $2 \mathrm{H}-1,44$ & 7.94 & 0.832 & 0.955 & 0.83 & 0.793 & 0.836 & 0.747 & 0.789 & 0.046 & 0.0038 \\
\hline $2 \mathrm{H}-1,120$ & 8.70 & 0.911 & 0.955 & 0.99 & 0.945 & & 0.903 & & & \\
\hline $2 \mathrm{H}-2,44$ & 9.44 & 0.989 & 0.955 & 0.87 & 0.831 & 0.888 & 0.800 & 0.852 & 0.036 & 0.0038 \\
\hline $2 \mathrm{H}-2,120$ & 10.20 & 1.068 & 0.955 & 0.93 & 0.888 & & 0.850 & & & \\
\hline $2 \mathrm{H}-3,44$ & 10.94 & 1.146 & 0.954 & 0.91 & 0.868 & 0.878 & 0.834 & 0.842 & 0.036 & 0.0025 \\
\hline $2 \mathrm{H}-3,120$ & 11.70 & 1.225 & 0.955 & 0.94 & 0.897 & & 0.862 & & & \\
\hline $2 \mathrm{H}-4,44$ & 12.44 & 1.303 & 0.955 & 0.91 & 0.869 & 0.883 & 0.846 & 0.854 & 0.029 & 0.0029 \\
\hline $2 \mathrm{H}-4,120$ & 13.20 & 1.412 & 0.287 & 0.92 & 0.643 & & 0.613 & & & \\
\hline $2 \mathrm{H}-5,44$ & 13.94 & 1.669 & 0.287 & 0.93 & 0.267 & 0.455 & 0.254 & 0.433 & 0.022 & 0.0019 \\
\hline $2 \mathrm{H}-5,120$ & 14.70 & 1.934 & 0.287 & 0.92 & 0.264 & & 0.252 & & & \\
\hline $2 \mathrm{H}-6,44$ & 15.44 & 2.192 & 0.287 & 0.89 & 0.256 & 0.260 & 0.246 & 0.249 & 0.011 & 0.0011 \\
\hline $2 \mathrm{H}-6,120$ & 16.20 & 2.457 & 0.287 & 0.91 & 0.261 & & 0.247 & & & \\
\hline $3 \mathrm{H}-1,46$ & 17.46 & 2.895 & 0.287 & 0.90 & 0.258 & 0.260 & 0.245 & 0.246 & 0.014 & 0.0009 \\
\hline
\end{tabular}


Table 3 (continued).

\begin{tabular}{|c|c|c|c|c|c|c|c|c|c|c|}
\hline $\begin{array}{l}\text { Core, section } \\
\text { interval }(\mathrm{cm})\end{array}$ & $\begin{array}{l}\text { Depth } \\
\text { (mbsf) }\end{array}$ & $\begin{array}{l}\text { Age } \\
(\mathrm{Ma})\end{array}$ & $\begin{array}{c}\text { LSR } \\
(\mathrm{cm} / \mathrm{k} . \mathrm{y} .)\end{array}$ & $\begin{array}{c}\text { DBD } \\
\left(\mathrm{g} / \mathrm{cm}^{3}\right)\end{array}$ & MAR & $\begin{array}{l}\text { MAR } \\
\text { (ave.) }\end{array}$ & CAR & $\begin{array}{l}\text { CAR } \\
\text { (ave.) }\end{array}$ & $\begin{array}{c}\text { NCAR } \\
\text { (ave.) }\end{array}$ & $\begin{array}{r}\text { EAR } \\
\text { (ave.) }\end{array}$ \\
\hline $3 \mathrm{H}-1,121$ & 18.21 & 3.157 & 0.287 & 0.88 & 0.253 & & 0.241 & & & \\
\hline $3 \mathrm{H}-2,45$ & 18.95 & 3.414 & 0.287 & 0.85 & 0.244 & 0.248 & 0.233 & 0.237 & 0.011 & 0.0007 \\
\hline $3 \mathrm{H}-2,121$ & 19.71 & 3.679 & 0.287 & 0.95 & 0.273 & & 0.259 & & & \\
\hline $3 \mathrm{H}-3,45$ & 20.45 & 3.937 & 0.287 & 0.92 & 0.264 & 0.268 & 0.257 & 0.258 & 0.010 & 0.0006 \\
\hline $3 \mathrm{H}-3,121$ & 21.21 & 4.201 & 0.287 & 0.94 & 0.270 & & 0.258 & & & \\
\hline $3 \mathrm{H}-4,45$ & 21.95 & 4.459 & 0.287 & 1.00 & 0.287 & 0.279 & 0.280 & 0.269 & 0.010 & 0.0009 \\
\hline $3 \mathrm{H}-4,121$ & 22.71 & 4.724 & 0.287 & 1.01 & 0.290 & & 0.281 & & & \\
\hline $3 \mathrm{H}-5,45$ & 23.45 & 4.982 & 0.287 & 1.02 & 0.293 & 0.291 & 0.282 & 0.281 & 0.010 & 0.0005 \\
\hline $3 \mathrm{H}-5,121$ & 24.21 & 5.246 & 0.287 & 0.93 & 0.267 & & 0.248 & & & \\
\hline $3 \mathrm{H}-6,45$ & 24.95 & 5.504 & 0.287 & 0.81 & 0.233 & 0.250 & 0.222 & 0.235 & 0.015 & 0.0002 \\
\hline $4 \mathrm{H}-1,120$ & 27.70 & 11.705 & 0.929 & 0.77 & 0.034 & & 0.032 & & & \\
\hline $4 \mathrm{H}-2,45$ & 28.45 & 11.785 & 0.929 & 0.75 & 0.697 & 0.366 & 0.653 & 0.341 & 0.024 & 0.0003 \\
\hline $4 \mathrm{H}-2,120$ & 29.20 & 11.866 & 0.929 & 0.77 & 0.716 & & 0.669 & & & \\
\hline $4 \mathrm{H}-3,45$ & 29.95 & 11.947 & 0.929 & 0.75 & 0.697 & 0.706 & 0.654 & 0.662 & 0.045 & 0.0004 \\
\hline $4 \mathrm{H}-3,120$ & 30.70 & 12.027 & 0.929 & 0.74 & 0.688 & & 0.619 & & & \\
\hline $4 \mathrm{H}-4,45$ & 31.45 & 12.108 & 0.929 & 0.75 & 0.697 & 0.692 & 0.633 & 0.626 & 0.066 & 0.0003 \\
\hline $4 \mathrm{H}-4,120$ & 32.20 & 12.189 & 0.929 & 0.72 & 0.669 & & 0.632 & & & \\
\hline $4 \mathrm{H}-5,45$ & 32.95 & 12.269 & 0.929 & 0.75 & 0.697 & 0.683 & 0.636 & 0.634 & 0.049 & 0.0003 \\
\hline $4 \mathrm{H}-5,120$ & 33.70 & 12.350 & 0.929 & 0.71 & 0.660 & & 0.612 & & & \\
\hline $5 \mathrm{H}-1,45$ & 36.45 & 12.646 & 0.929 & 0.69 & 0.641 & 0.651 & 0.605 & 0.609 & 0.042 & 0.0003 \\
\hline $5 \mathrm{H}-1,120$ & 37.20 & 12.727 & 0.929 & 0.68 & 0.632 & & 0.602 & & & \\
\hline $5 \mathrm{H}-2,45$ & 37.95 & 12.807 & 0.929 & 0.65 & 0.604 & 0.618 & 0.571 & 0.586 & 0.032 & 0.0002 \\
\hline $5 \mathrm{H}-2,120$ & 38.70 & 12.888 & 0.929 & 0.67 & 0.623 & & 0.578 & & & \\
\hline $5 \mathrm{H}-3,45$ & 39.45 & 12.969 & 0.929 & 0.80 & 0.743 & 0.683 & 0.700 & 0.639 & 0.044 & 0.0002 \\
\hline $5 \mathrm{H}-3,120$ & 40.20 & 13.050 & 0.929 & 0.79 & 0.734 & & 0.688 & & & \\
\hline $5 \mathrm{H}-4,45$ & 40.95 & 13.130 & 0.929 & 0.79 & 0.734 & 0.734 & 0.698 & 0.693 & 0.041 & 0.0002 \\
\hline $5 \mathrm{H}-4,120$ & 41.70 & 13.211 & 0.929 & 0.77 & 0.716 & & 0.685 & & & \\
\hline $6 \mathrm{H}-1,44$ & 45.94 & 13.667 & 0.929 & 0.74 & 0.688 & 0.702 & 0.642 & 0.663 & 0.038 & 0.0003 \\
\hline $6 \mathrm{H}-1,120$ & 46.70 & 13.749 & 0.929 & 0.77 & 0.715 & & 0.685 & & & \\
\hline $6 \mathrm{H}-2,44$ & 47.44 & 13.829 & 0.930 & 0.76 & 0.707 & 0.711 & 0.677 & 0.681 & 0.030 & 0.0004 \\
\hline $6 \mathrm{H}-2,120$ & 48.20 & 13.910 & 0.929 & 0.77 & 0.715 & & 0.674 & & & \\
\hline $6 \mathrm{H}-3,44$ & 48.94 & 13.990 & 0.930 & 0.76 & 0.707 & 0.711 & 0.671 & 0.672 & 0.039 & 0.0003 \\
\hline $6 \mathrm{H}-3,120$ & 49.70 & 14.072 & 0.929 & 0.81 & 0.753 & & 0.710 & & & \\
\hline $6 \mathrm{H}-4,44$ & 50.44 & 14.151 & 0.930 & 0.78 & 0.725 & 0.739 & 0.693 & 0.702 & 0.037 & 0.0006 \\
\hline $6 \mathrm{H}-4,120$ & 51.20 & 14.233 & 0.929 & 0.77 & 0.715 & & 0.678 & & & \\
\hline $6 \mathrm{H}-5,44$ & 51.94 & 14.313 & 0.930 & 0.78 & 0.725 & 0.720 & 0.689 & 0.683 & 0.037 & \\
\hline $6 \mathrm{H}-5,120$ & 52.70 & 14.395 & 0.929 & 0.80 & 0.743 & & 0.677 & & & \\
\hline $6 \mathrm{H}-6,44$ & 53.44 & 14.437 & 1.733 & 0.79 & 1.369 & 1.056 & 1.322 & 0.991 & 0.066 & 0.0007 \\
\hline
\end{tabular}

Notes: $\mathrm{LSR}=$ linear sedimentation rates $(\mathrm{cm} / \mathrm{k} \cdot \mathrm{y}$.), $\mathrm{DBD}=$ dry bulk density, $\mathrm{MAR}=$ mass (bulk) sediment accumulation rate, $\mathrm{CAR}=$ carbonate accumulation rate, $\mathrm{NCAR}=$ noncarbonate accumuation rate, and EAR $=$ eolian (e.g., fine-grained lithogenic material, $<63 \mu \mathrm{m}$ in size) accumulation rate. Accumulation rates are given in units of g/cm ${ }^{2} / \mathrm{k} . \mathrm{y}$. Average values are used when two successive samples were used to conduct the chemical extraction procedure. 
Table 4. Calcium carbonate determinations, Holes $872 \mathrm{~A}$ and $872 \mathrm{C}$.

\begin{tabular}{|c|c|c|c|c|c|}
\hline $\begin{array}{l}\text { Core, section, } \\
\text { interval }(\mathrm{cm})\end{array}$ & $\begin{array}{l}\text { Depth } \\
\text { (mbsf) }\end{array}$ & $\begin{array}{c}\text { Carbonate } \\
(w t \%)\end{array}$ & $\begin{array}{l}\text { Core, section, } \\
\text { interval }(\mathrm{cm})\end{array}$ & $\begin{array}{l}\text { Depth } \\
\text { (mbsf) }\end{array}$ & $\begin{array}{c}\text { Carbonate } \\
\text { (wt\%) }\end{array}$ \\
\hline \multicolumn{3}{|l|}{$144-872 \mathrm{~A}-$} & $1 \mathrm{H}-4,45-47$ & 4.16 & 94.41 \\
\hline IH-1, $45-47$ & 0.45 & 94.61 & $1 \mathrm{H}-4,118-120$ & 4.89 & 94.69 \\
\hline $1 \mathrm{H}-1,120-122$ & 1.20 & 94.34 & $1 \mathrm{H}-5,45-47$ & 5.55 & 90.09 \\
\hline $1 \mathrm{H}-2,45-47$ & 1.95 & 94.23 & IH $-5,118-120$ & 6.28 & 93.71 \\
\hline $1 \mathrm{H}-2,120-122$ & 2.70 & 92.43 & $1 \mathrm{H}-6,45-47$ & 6.93 & 94.19 \\
\hline IH- $3,45-47$ & 3.45 & 93.53 & $2 \mathrm{H}-1,45-47$ & 9.45 & 94.47 \\
\hline $1 \mathrm{H}-3,120-122$ & 4.20 & 93.91 & $2 \mathrm{H}-2,45-47$ & 10.41 & 95.76 \\
\hline IH $-4,45-47$ & 4.95 & 94.63 & $2 \mathrm{H}-2,119-121$ & 11.15 & 94.20 \\
\hline $1 \mathrm{H}-4,120-122$ & 5.70 & 94.59 & $2 \mathrm{H}-3,45-47$ & 11.77 & 94.90 \\
\hline IH $-5,45-47$ & 6.45 & 95.56 & $2 \mathrm{H}-3,119-121$ & 12.51 & 91.79 \\
\hline 1H-5, $120-122$ & 7.20 & 96.47 & $2 \mathrm{H}-4,45-47$ & 13.15 & 94.79 \\
\hline $2 \mathrm{H}-1,45-46$ & 7.95 & 94.28 & $2 \mathrm{H}-4,119-12 \mathrm{I}$ & 13.89 & 95.11 \\
\hline $2 \mathrm{H}-1,120-121$ & 8.70 & 95.06 & $2 \mathrm{H}-5,45-47$ & 14.47 & 94.62 \\
\hline $2 \mathrm{H}-2,45-46$ & 9.45 & 95.06 & $2 \mathrm{H}-5,119-121$ & 15.21 & 95.36 \\
\hline $2 \mathrm{H}-2,120-121$ & 10.20 & 95.47 & $2 \mathrm{H}-6,45-47$ & 15.84 & 90.14 \\
\hline $2 \mathrm{H}-3,45-46$ & 10.95 & 96.01 & $2 \mathrm{H}-6,119-121$ & 16.58 & 92.89 \\
\hline $2 \mathrm{H}-3,120-121$ & 11.70 & 95.25 & $3 \mathrm{H}-1,45-47$ & 18.95 & 94.04 \\
\hline $2 \mathrm{H}-4,45-46$ & 12.45 & 96.15 & $3 \mathrm{H}-1,97-99$ & 19.47 & 93.44 \\
\hline $2 \mathrm{H}-4,120-122$ & 13.20 & 95.99 & $3 \mathrm{H}-2,45-47$ & 20.01 & 92.58 \\
\hline $2 \mathrm{H}-5,45-46$ & 13.95 & 95.10 & $3 \mathrm{H}-2,11 \mathrm{I}-113$ & 20.67 & 93.13 \\
\hline $2 \mathrm{H}-5,120-122$ & 14.70 & 94.87 & $3 \mathrm{H}-3,45-47$ & 21.22 & 94.26 \\
\hline $2 \mathrm{H}-6,44-46$ & 15.44 & 95.75 & $3 \mathrm{H}-3,116-118$ & 21.93 & 92.86 \\
\hline $4 \mathrm{H}-1,45-47$ & 26.95 & 95.44 & $3 \mathrm{H}-4,45-47$ & 22.52 & 92.60 \\
\hline $4 \mathrm{H}-1,119-121$ & 27.69 & 95.02 & $3 \mathrm{H}-4,115-117$ & 23.22 & 94.00 \\
\hline $4 \mathrm{H}-2,45-47$ & 28.45 & 95.53 & $3 \mathrm{H}-5,45-47$ & 23.83 & 92.09 \\
\hline $4 \mathrm{H}-2,119-121$ & 29.19 & 93.63 & $3 \mathrm{H}-5,115-117$ & 24.53 & 93.67 \\
\hline $4 \mathrm{H}-3,45-47$ & 29.95 & 95.01 & $3 \mathrm{H}-6,45-47$ & 25.15 & 92.52 \\
\hline $4 \mathrm{H}-3,119-121$ & 30.69 & 96.08 & $3 \mathrm{H}-6,117-119$ & 25.87 & 94.12 \\
\hline $4 \mathrm{H}-4,45-47$ & 31.45 & 94.41 & $4 \mathrm{H}-1,45-47$ & 28.45 & 93.49 \\
\hline $4 \mathrm{H}-4,119-121$ & 32.19 & 94.42 & $4 \mathrm{H}-2,45-47$ & 29.47 & 94.94 \\
\hline $4 \mathrm{H}-5,45-47$ & 32.95 & 93.21 & $4 \mathrm{H}-2,113-115$ & 30.15 & 91.57 \\
\hline $4 \mathrm{H}-5,119-121$ & 33.69 & 95.55 & $4 \mathrm{H}-3,45-47$ & 30.73 & 95.15 \\
\hline $5 \mathrm{H}-1,44-46$ & 36.44 & 94.58 & $4 \mathrm{H}-3,113-115$ & 31.41 & 92.65 \\
\hline $5 \mathrm{H}-1,119-121$ & 37.19 & 94.95 & $4 \mathrm{H}-4,45-47$ & 32.06 & 95.34 \\
\hline $5 \mathrm{H}-2,44-46$ & 37.94 & 95.51 & $4 \mathrm{H}-4,113-115$ & 32.74 & 93.48 \\
\hline $5 \mathrm{H}-2,119-121$ & 38.69 & 95.78 & $4 \mathrm{H}-5,45-47$ & 33.36 & 94.40 \\
\hline $5 \mathrm{H}-3,44-46$ & 39.44 & 95.04 & $4 \mathrm{H}-5,113-115$ & 34.04 & 92.93 \\
\hline $5 \mathrm{H}-3,119-121$ & 40.19 & 97.07 & $4 \mathrm{H}-6,45-47$ & 34.57 & 94.14 \\
\hline $5 \mathrm{H}-4,44-46$ & 40.94 & 95.06 & $4 \mathrm{H}-6,113-115$ & 35.25 & 93.82 \\
\hline $5 \mathrm{H}-4,119-121$ & 41.69 & 95.00 & $5 \mathrm{H}-1,45-47$ & 37.95 & 94.38 \\
\hline $5 \mathrm{H}-5,44-46$ & 42.44 & 93.48 & $5 \mathrm{H}-2,45-47$ & 38.81 & 94.27 \\
\hline $5 \mathrm{H}-5,119-121$ & 43.19 & 95.24 & $5 \mathrm{H}-3,45-47$ & 39.96 & 93.99 \\
\hline $5 \mathrm{H}-6,44-46$ & 43.94 & 96.66 & $5 \mathrm{H}-3,116-118$ & 40.67 & 89.94 \\
\hline $6 \mathrm{H}-\mathrm{I}, 45-47$ & 45.95 & 91.81 & $5 \mathrm{H}-4,45-47$ & 41.22 & 93.31 \\
\hline $6 \mathrm{H}-1,119-121$ & 46.69 & 94.40 & $5 \mathrm{H}-4,116-118$ & 41.93 & 93.72 \\
\hline $6 \mathrm{H}-2,45-47$ & 47.45 & 92.45 & $5 \mathrm{H}-5,45-47$ & 42.41 & 91.64 \\
\hline $6 \mathrm{H}-2,119-121$ & 48.19 & 94.82 & $5 \mathrm{H}-5,116-118$ & 43.12 & 93.93 \\
\hline $6 \mathrm{H}-3,45-47$ & 48.95 & 92.93 & $5 \mathrm{H}-6,45-47$ & 43.61 & 95.95 \\
\hline $6 \mathrm{H}-3,119-121$ & 49.69 & 93.35 & $6 \mathrm{H}-1,30-32$ & 47.30 & 94.43 \\
\hline $6 \mathrm{H}-4,45-47$ & 50.45 & 93.90 & $6 \mathrm{H}-2,30-32$ & 48.19 & 93.27 \\
\hline $6 \mathrm{H}-4,119-121$ & 51.19 & 93.04 & $6 \mathrm{H}-2,105-107$ & 48.94 & 93.51 \\
\hline $6 \mathrm{H}-5,45-47$ & 51.95 & 90.88 & $6 \mathrm{H}-3,30-32$ & 49.45 & 91.00 \\
\hline $6 \mathrm{H}-5,119-121$ & 52.69 & 94.97 & $6 \mathrm{H}-3,105-107$ & 50.20 & 92.14 \\
\hline $6 \mathrm{H}-6,45-47$ & 53.45 & 93.70 & $6 \mathrm{H}-4,30-32$ & 50.74 & 94.36 \\
\hline $144-872 \mathrm{C}$ - & & & $6 \mathrm{H}-4,105-107$ & 51.49 & 92.93 \\
\hline $1 \mathrm{H}-1,45-47$ & 0.45 & 94.20 & $6 \mathrm{H}-5,30-32$ & 52.01 & 94.10 \\
\hline IH- $2,45-47$ & 1.45 & 93.51 & $6 \mathrm{H}-5,105-107$ & 52.76 & 93.20 \\
\hline $1 \mathrm{H}-2,118-120$ & 2.18 & 90.94 & $6 \mathrm{H}-6,30-32$ & 53.32 & 92.77 \\
\hline $\begin{array}{l}1 \mathrm{H}-3,45-47 \\
1 \mathrm{H}-3,118-120\end{array}$ & $\begin{array}{l}2.75 \\
3.48\end{array}$ & $\begin{array}{l}95.61 \\
95.07\end{array}$ & nate $=$ & & cit \\
\hline
\end{tabular}

Table 5. Age models for Hole 872A.

\begin{tabular}{|c|c|c|c|c|c|}
\hline $\begin{array}{l}\text { Depth } \\
\text { top } \\
\text { (mbsf) }\end{array}$ & $\begin{array}{l}\text { Depth } \\
\text { bottom } \\
\text { (mbsf) }\end{array}$ & $\begin{array}{l}\text { Depth } \\
\text { middle } \\
\text { (mbsf) }\end{array}$ & $\begin{array}{l}\text { Age } \\
(\mathrm{Ma})\end{array}$ & Code & Datum \\
\hline 0.00 & 0.00 & 0.00 & 0.000 & & \\
\hline 0.85 & 1.35 & 1.10 & 0.280 & $\mathrm{Cl}$ & FAD E huxleyi \\
\hline 1.75 & 2.35 & 2.05 & 0.470 & $\mathrm{C} 2$ & LAD P. lacunosa \\
\hline 5.85 & 6.25 & 6.05 & 1.370 & $\mathrm{C} 3$ & LAD H. sellii** \\
\hline 7.50 & 7.50 & 7.50 & 1.450 & $\mathrm{C} 4$ & LAD C. macinteyrei \\
\hline 7.50 & 7.75 & 7.62 & 1.900 & $\mathrm{C} 5$ & LAD D. brouweri \\
\hline 17.00 & 30.09 & 23.55 & 5.200 & F4 & FAD $G$. tumida \\
\hline 30.09 & 36.00 & 33.04 & 5.800 & F5 & FAD P. primalis** \\
\hline 36.00 & 45.50 & 40.75 & 8.850 & C7 & LAD D. hamatus \\
\hline 36.00 & 45.50 & 40.75 & 9.000 & C8 & LAD C. coalitus \\
\hline 45.50 & 46.00 & 45.75 & 10.000 & $\mathrm{C} 9$ & FAD D. hamatus \\
\hline 47.00 & 47.50 & 47.25 & 10.800 & $\mathrm{C} 10$ & FAD C coalitus \\
\hline 57.09 & 57.72 & 57.40 & 11.500 & F6 & LAD G. fohsi lobata** \\
\hline 68.00 & 70.00 & 69.00 & 14.400 & $\mathrm{C} 11$ & LAD S. heteromorphus** \\
\hline
\end{tabular}

Notes: Age models were generated using the midpoint depth (mbsf) for each datum. Double asterisks $\left({ }^{* *}\right)$ indicate the datums that were used to generate the "simplified" version of the age model. FAD $=$ first appearance datum, and $\mathrm{LAD}=$ last appearance datum. Codes are taken from Premoli Silva, Haggerty, Rack, et al. (1993). $\mathrm{C}=$ nannofossil datum, and $\mathrm{F}=$ foraminifer datum. 
Table 6. Linear sedimentation and mass accumulation rates for Hole 872A, calculated using the comprehensive and simplified age models.

\begin{tabular}{|c|c|c|c|c|c|c|c|}
\hline $\begin{array}{l}\text { Core, section, } \\
\text { interval }(\mathrm{cm})\end{array}$ & $\begin{array}{l}\text { Depth } \\
\text { (mbsf) }\end{array}$ & $\begin{array}{l}\text { Age } \\
\text { (Ma) }\end{array}$ & $\begin{array}{c}\text { LSR } \\
(\mathrm{cm} / \mathrm{k} \cdot \mathrm{y} .)\end{array}$ & $\begin{array}{c}\text { DBD } \\
\left(\mathrm{g} / \mathrm{cm}^{3}\right)\end{array}$ & MAR & CAR & NCAR \\
\hline \multicolumn{8}{|c|}{ Comprehensive model: } \\
\hline $1 \mathrm{H}-1,45$ & 0.45 & 0.11 & 0.393 & 0.86 & 0.338 & 0.320 & 0.018 \\
\hline $1 \mathrm{H}-1,120$ & 1.20 & 0.30 & 0.500 & 0.79 & 0.395 & 0.373 & 0.022 \\
\hline $1 \mathrm{H}-2,45$ & 1.95 & 0.45 & 0.500 & 0.85 & 0.425 & 0.400 & 0.025 \\
\hline $\mathrm{IH}-2,120$ & 2.70 & 0.62 & 0.444 & 0.93 & 0.413 & 0.382 & 0.031 \\
\hline IH $-3,45$ & 3.45 & 0.79 & 0.444 & 0.92 & 0.408 & 0.382 & 0.026 \\
\hline $1 \mathrm{H}-3,120$ & 4.20 & 0.95 & 0.444 & 0.94 & 0.417 & 0.392 & 0.025 \\
\hline IH $-4,45$ & 4.95 & 1.12 & 0.444 & 0.95 & 0.422 & 0.399 & 0.023 \\
\hline $1 \mathrm{H}-4,120$ & 5.70 & 1.29 & 0.444 & 0.93 & 0.413 & 0.391 & 0.022 \\
\hline IH $-5,45$ & 6.45 & 1.39 & 1.813 & 0.93 & 1.686 & 1.611 & 0.075 \\
\hline IH-5, 120 & 7.20 & 1.43 & 1.813 & 0.94 & 1.704 & 1.644 & 0.060 \\
\hline $2 \mathrm{H}-1,45$ & 7.95 & 1.99 & 0.483 & 0.95 & 0.459 & 0.433 & 0.026 \\
\hline $2 \mathrm{H}-1,120$ & 8.70 & 2.18 & 0.382 & 0.95 & 0.363 & 0.345 & 0.018 \\
\hline $2 \mathrm{H}-2,45$ & 9.45 & 2.38 & 0.382 & 0.94 & 0.359 & 0.341 & 0.018 \\
\hline $2 \mathrm{H}-2,120$ & 10.20 & 2.58 & 0.382 & 0.89 & 0.340 & 0.324 & 0.015 \\
\hline $2 \mathrm{H}-3,45$ & 10.95 & 2.77 & 0.382 & 0.89 & 0.340 & 0.326 & 0.014 \\
\hline $2 \mathrm{H}-3,120$ & 11.70 & 2.97 & 0.382 & 0.89 & 0.340 & 0.324 & 0.016 \\
\hline $2 \mathrm{H}-4,45$ & 12.45 & 3.16 & 0.382 & 0.90 & 0.344 & 0.330 & 0.013 \\
\hline $2 \mathrm{H}-4,120$ & 13.20 & 3.36 & 0.382 & 0.87 & 0.332 & 0.319 & 0.013 \\
\hline $2 \mathrm{H}-5,45$ & 13.95 & 3.56 & 0.382 & 0.88 & 0.336 & 0.320 & 0.016 \\
\hline $2 \mathrm{H}-5,120$ & 14.70 & 3.75 & 0.382 & 0.90 & 0.344 & 0.326 & 0.018 \\
\hline $2 \mathrm{H}-6,44$ & 15.44 & 3.95 & 0.382 & 0.90 & 0.344 & 0.329 & 0.015 \\
\hline $4 \mathrm{H}-1,45$ & 26.95 & 5.41 & 1.582 & 0.92 & 1.455 & 1.389 & 0.066 \\
\hline $4 \mathrm{H}-1,119$ & 27.69 & 5.46 & 1.582 & 0.95 & 1.503 & 1.428 & 0.075 \\
\hline $4 \mathrm{H}-2,45$ & 28.45 & 5.51 & 1.582 & 1.11 & 1.756 & 1.678 & 0.078 \\
\hline $4 \mathrm{H}-2,119$ & 29.19 & 5.56 & 1.582 & 1.04 & 1.645 & 1.540 & 0.105 \\
\hline $4 \mathrm{H}-3,45$ & 29.95 & 5.60 & 1.582 & 1.06 & 1.677 & 1.593 & 0.084 \\
\hline $4 \mathrm{H}-3,119$ & 30.69 & 5.65 & 1.582 & 0.88 & 1.392 & 1.338 & 0.055 \\
\hline $4 \mathrm{H}-4,45$ & 31.45 & 5.70 & 1.582 & 0.83 & 1.313 & 1.240 & 0.073 \\
\hline $4 \mathrm{H}-4,119$ & 32.19 & 5.75 & 1.582 & 0.83 & 1.313 & 1.240 & 0.073 \\
\hline $4 \mathrm{H}-5,45$ & 32.95 & 5.79 & 1.582 & 0.84 & 1.329 & 1.239 & 0.090 \\
\hline $4 \mathrm{H}-5,119$ & 33.69 & 6.06 & 0.253 & 0.78 & 0.197 & 0.189 & 0.009 \\
\hline $5 \mathrm{H}-1,44$ & 36.44 & 7.14 & 0.253 & 0.77 & 0.195 & 0.184 & 0.011 \\
\hline $5 \mathrm{H}-1,119$ & 37.19 & 7.44 & 0.253 & 0.76 & 0.192 & 0.182 & 0.010 \\
\hline $5 \mathrm{H}-2,44$ & 37.94 & 7.74 & 0.253 & 0.75 & 0.190 & 0.181 & 0.009 \\
\hline $5 \mathrm{H}-2,119$ & 38.69 & 8.04 & 0.253 & 0.75 & 0.190 & 0.182 & 0.008 \\
\hline $5 \mathrm{H}-3,44$ & 39.44 & 8.33 & 0.253 & 0.73 & 0.185 & 0.175 & 0.009 \\
\hline $5 \mathrm{H}-3,119$ & 40.19 & 8.63 & 0.253 & 0.72 & 0.182 & 0.177 & 0.005 \\
\hline $5 \mathrm{H}-4,44$ & 40.94 & 9.04 & 0.500 & 0.72 & 0.360 & 0.342 & 0.018 \\
\hline $5 \mathrm{H}-4,119$ & 41.69 & 9.19 & 0.500 & 0.72 & 0.360 & 0.342 & 0.018 \\
\hline $5 \mathrm{H}-5,44$ & 42.44 & 9.34 & 0.500 & 0.73 & 0.365 & 0.341 & 0.024 \\
\hline $5 \mathrm{H}-5,119$ & 43.19 & 9.49 & 0.500 & 0.73 & 0.365 & 0.348 & 0.017 \\
\hline $5 \mathrm{H}-6,44$ & 43.94 & 9.64 & 0.500 & 0.73 & 0.365 & 0.353 & 0.012 \\
\hline $6 \mathrm{H}-1,45$ & 45.95 & 10.11 & 0.188 & 0.73 & 0.137 & 0.126 & 0.011 \\
\hline $6 \mathrm{H}-1,119$ & 46.69 & 10.50 & 0.188 & 0.70 & 0.131 & 0.124 & 0.007 \\
\hline $6 \mathrm{H}-2,45$ & 47.45 & 10.81 & 1.451 & 0.69 & 1.001 & 0.926 & 0.076 \\
\hline $6 \mathrm{H}-2,119$ & 48.19 & 10.86 & 1.451 & 0.68 & 0.987 & 0.936 & 0.051 \\
\hline $6 \mathrm{H}-3,45$ & 48.95 & 10.92 & 1.451 & 0.68 & 0.987 & 0.917 & 0.070 \\
\hline $6 \mathrm{H}-3,119$ & 49.69 & 10.97 & 1.451 & 0.68 & 0.987 & 0.921 & 0.066 \\
\hline $6 \mathrm{H}-4,45$ & 50.45 & 11.02 & 1.451 & 0.69 & 1.001 & 0.940 & 0.061 \\
\hline $6 \mathrm{H}-4,119$ & 51.19 & 11.07 & 1.451 & 0.71 & 1.030 & 0.958 & 0.072 \\
\hline $6 \mathrm{H}-5,45$ & 51.95 & 11.12 & 1.451 & 0.71 & 1.030 & 0.936 & 0.094 \\
\hline $6 \mathrm{H}-5,119$ & 52.69 & 11.18 & 1.451 & $0.7 i$ & 1.030 & 0.978 & 0.052 \\
\hline $6 \mathrm{H}-6,45$ & 53.45 & 11.23 & 1.451 & 0.72 & 1.045 & 0.979 & 0.066 \\
\hline \multicolumn{8}{|l|}{ Simplified model: } \\
\hline IH- 1,45 & 0.45 & 0.102 & 0.442 & 0.8 & 0.380 & 0.359 & 0.021 \\
\hline $1 \mathrm{H}-1,120$ & 1.20 & 0.272 & 0.442 & 0.79 & 0.349 & 0.329 & 0.020 \\
\hline
\end{tabular}

\begin{tabular}{|c|c|c|c|c|c|c|c|}
\hline $\begin{array}{l}\text { Core, section, } \\
\text { interval }(\mathrm{cm})\end{array}$ & $\begin{array}{l}\text { Depth } \\
\text { (mbsf) }\end{array}$ & $\begin{array}{l}\text { Age } \\
\text { (Ma) }\end{array}$ & $\begin{array}{c}\text { LSR } \\
\left(\mathrm{cm} / \mathrm{k} . \mathrm{y}_{*}\right)\end{array}$ & $\underset{\left(\mathrm{g} / \mathrm{cm}^{3}\right)}{\text { DBD }}$ & MAR & CAR & NCAR \\
\hline $1 \mathrm{H}-2,45$ & 1.95 & 0.442 & 0.442 & 0.85 & 0.375 & 0.354 & 0.021 \\
\hline $1 \mathrm{H}-2,120$ & 2.70 & 0.611 & 0.442 & 0.93 & 0.411 & 0.380 & 0.031 \\
\hline I H $-3,45$ & 3.45 & 0.781 & 0.442 & 0.92 & 0.406 & 0.380 & 0.026 \\
\hline $1 \mathrm{H}-3,120$ & 4.20 & 0.951 & 0.442 & 0.94 & 0.415 & 0.390 & 0.025 \\
\hline IH $-4,45$ & 4.95 & 1.121 & 0.442 & 0.95 & 0.420 & 0.397 & 0.023 \\
\hline $1 \mathrm{H}-4,120$ & 5.70 & 1.291 & 0.442 & 0.93 & 0.411 & 0.389 & 0.022 \\
\hline IH- 5,45 & 6.45 & 1.436 & 0.609 & 0.93 & 0.481 & 0.460 & 0.021 \\
\hline $1 \mathrm{H}-5,120$ & 7.20 & 1.559 & 0.609 & 0.94 & 0.573 & 0.552 & 0.020 \\
\hline $2 \mathrm{H}-1,45$ & 7.95 & 1.682 & 0.609 & 0.95 & 0.579 & 0.546 & 0.033 \\
\hline $2 \mathrm{H}-1,120$ & 8.70 & 1.805 & 0.609 & 0.95 & 0.579 & 0.550 & 0.029 \\
\hline $2 \mathrm{H}-2,45$ & 9.45 & 1.928 & 0.609 & 0.94 & 0.573 & 0.544 & 0.028 \\
\hline $2 \mathrm{H}-2,120$ & 10.20 & 2.051 & 0.609 & 0.89 & 0.542 & 0.518 & 0.025 \\
\hline $2 \mathrm{H}-3,45$ & 10.95 & 2.174 & 0.609 & 0.89 & 0.542 & 0.521 & 0.022 \\
\hline $2 \mathrm{H}-3,120$ & 11.70 & 2.297 & 0.609 & 0.89 & 0.542 & 0.516 & 0.026 \\
\hline $2 \mathrm{H}-4,45$ & 12.45 & 2.421 & 0.609 & 0.90 & 0.548 & 0.527 & 0.021 \\
\hline $2 \mathrm{H}-4,120$ & 13.20 & 2.544 & 0.609 & 0.87 & 0.530 & 0.509 & 0.021 \\
\hline $2 \mathrm{H}-5,45$ & 13.95 & 2.667 & 0.609 & 0.88 & 0.536 & 0.510 & 0.026 \\
\hline $2 \mathrm{H}-5,120$ & 14.70 & 2.790 & 0.609 & 0.90 & 0.548 & 0.520 & 0.028 \\
\hline $2 \mathrm{H}-6,44$ & 15.44 & 2.911 & 0.609 & 0.90 & 0.549 & 0.525 & 0.023 \\
\hline $4 \mathrm{H}-1,45$ & 26.95 & 4.800 & 0.609 & 0.92 & 0.561 & 0.535 & 0.026 \\
\hline $4 \mathrm{H}-1,119$ & 27.69 & 4.922 & 0.609 & 0.95 & 0.579 & 0.550 & 0.029 \\
\hline $4 \mathrm{H}-2,45$ & 28.45 & 5.047 & 0.609 & 1.11 & 0.677 & 0.646 & 0.030 \\
\hline $4 \mathrm{H}-2,119$ & 29.19 & 5.168 & 0.609 & 1.04 & 0.633 & 0.593 & 0.040 \\
\hline $4 \mathrm{H}-3,45$ & 29.95 & 5.293 & 0.609 & 1.06 & 0.646 & 0.614 & 0.032 \\
\hline $4 \mathrm{H}-3,119$ & 30.69 & 5.414 & 0.609 & 0.88 & 0.536 & 0.515 & 0.021 \\
\hline $4 \mathrm{H}-4,45$ & 31.45 & 5.539 & 0.609 & 0.83 & 0.506 & 0.478 & 0.028 \\
\hline $4 \mathrm{H}-4,119$ & 32.19 & 5.661 & 0.609 & 0.83 & 0.506 & 0.477 & 0.028 \\
\hline $4 \mathrm{H}-5,45$ & 32.95 & 5.785 & 0.609 & 0.84 & 0.512 & 0.477 & 0.035 \\
\hline $4 \mathrm{H}-5,119$ & 33.69 & 5.956 & 0.418 & 0.78 & 0.339 & 0.324 & 0.015 \\
\hline $5 \mathrm{H}-1,44$ & 36.44 & 6.613 & 0.418 & 0.77 & 0.322 & 0.305 & 0.017 \\
\hline $5 \mathrm{H}-1,119$ & 37.19 & 6.793 & 0.418 & 0.76 & 0.318 & 0.302 & 0.016 \\
\hline $5 \mathrm{H}-2,44$ & 37.94 & 6.972 & 0.418 & 0.75 & 0.314 & 0.299 & 0.014 \\
\hline $5 \mathrm{H}-2,119$ & 38.69 & 7.151 & 0.418 & 0.75 & 0.314 & 0.300 & 0.013 \\
\hline $5 \mathrm{H}-3,44$ & 39.44 & 7.331 & 0.418 & 0.73 & 0.305 & 0.290 & 0.015 \\
\hline $5 \mathrm{H}-3,119$ & 40.19 & 7.510 & 0.418 & 0.72 & 0.301 & 0.292 & 0.009 \\
\hline $5 \mathrm{H}-4,44$ & 40.94 & 7.689 & 0.418 & 0.72 & 0.301 & 0.286 & 0.015 \\
\hline $5 \mathrm{H}-4,119$ & 41.69 & 7.869 & 0.418 & 0.72 & 0.301 & 0.286 & 0.015 \\
\hline SH $-5,44$ & 42.44 & 8.048 & 0.418 & 0.73 & 0.305 & 0.285 & 0.020 \\
\hline $5 \mathrm{H}-5,119$ & 43.19 & 8.227 & 0.418 & 0.73 & 0.305 & 0.291 & 0.015 \\
\hline 5H-6, 44 & 43.94 & 8.407 & 0.418 & 0.73 & 0.305 & 0.295 & 0.010 \\
\hline $6 \mathrm{H}-1,45$ & 45.95 & 8.887 & 0.418 & 0.73 & 0.305 & 0.280 & 0.025 \\
\hline $6 \mathrm{H}-1,119$ & 46.69 & 9.064 & 0.418 & 0.70 & 0.293 & 0.276 & 0.016 \\
\hline $6 \mathrm{H}-2,45$ & 47.45 & 9.246 & 0.418 & 0.69 & 0.289 & 0.267 & 0.022 \\
\hline $6 \mathrm{H}-2,119$ & 48.19 & 9.423 & 0.418 & 0.68 & 0.284 & 0.270 & 0.015 \\
\hline $6 \mathrm{H}-3,45$ & 48.95 & 9.605 & 0.418 & 0.68 & 0.284 & 0.264 & 0.020 \\
\hline $6 \mathrm{H}-3,119$ & 49.69 & 9.782 & 0.418 & 0.68 & 0.284 & 0.265 & 0.019 \\
\hline $6 \mathrm{H}-4,45$ & 50.45 & 9.964 & 0.418 & 0.69 & 0.288 & 0.271 & 0.018 \\
\hline $6 \mathrm{H}-4,119$ & 51.19 & 10.141 & 0.418 & 0.71 & 0.297 & 0.276 & 0.021 \\
\hline $6 \mathrm{H}-5,45$ & 51.95 & 10.322 & 0.418 & 0.71 & 0.297 & 0.270 & 0.027 \\
\hline $6 \mathrm{H}-5,119$ & 52.69 & 10.499 & 0.418 & 0.71 & 0.297 & 0.282 & 0.015 \\
\hline $6 \mathrm{H}-6,45$ & 53.45 & 10.681 & 0.418 & 0.72 & 0.301 & 0.282 & 0.019 \\
\hline
\end{tabular}

Notes: $\mathrm{LSR}=$ linear sedimentation rates $(\mathrm{cm} / \mathrm{k}, \mathrm{y}),. \mathrm{DBD}=$ dry bulk density, MAR $=$ mass (bulk) sediment accumulation rate, $\mathrm{CAR}=$ carbonate accumulation rate, $\mathrm{NCAR}=$ noncarbonate accumuation rate, and EAR = eolian (e.g., fine-grained lithogenic material, $<63 \mu \mathrm{m}$ in size) accumulation rate. Accumulation rates are given in units of $\mathrm{g} / \mathrm{cm}^{2} / \mathrm{k}$.y. Average values are used when two successive samples were used to conduct the chemical extraction procedure. 
Table 7. Calcium carbonate determinations and chemical extractions of fine-grained lithogenic material, Hole 873B.

\begin{tabular}{|c|c|c|c|c|c|c|}
\hline $\begin{array}{l}\text { Core, section, } \\
\text { interval }(\mathrm{cm})\end{array}$ & $\begin{array}{l}\text { Depth } \\
\text { (mbsf) }\end{array}$ & $\begin{array}{l}\mathrm{CaCO}_{3} \\
\text { (wt\%) }\end{array}$ & $\begin{array}{l}\mathrm{CaCO}_{3} \\
\text { (ave.) } \\
\text { (wt } \% \text { ) }\end{array}$ & $\begin{array}{c}\text { Noncarbonate } \\
\text { (ave.) } \\
\text { (wt\%) }\end{array}$ & $\begin{array}{l}\text { Eolian } \\
\text { (ave.) } \\
\text { (wt\%) }\end{array}$ & $\begin{array}{l}\text { \%Eolian } \\
\text { carb-free }\end{array}$ \\
\hline \multicolumn{7}{|l|}{ 144-873B- } \\
\hline $1 \mathrm{H}-1,60-62$ & 0.60 & 91.31 & & & & \\
\hline $1 \mathrm{H}-1.117-119$ & 1.17 & 93.94 & 92.62 & 7.37 & 0.43 & 5.9 \\
\hline $1 \mathrm{H}-2,18-22$ & 1.37 & 92.82 & & & & \\
\hline $1 \mathrm{H}-2,60-62$ & 1.79 & 93.93 & 93.38 & 6.62 & 0.33 & 5.0 \\
\hline $1 \mathrm{H}-2,118-122$ & 2.37 & 95.34 & & & & \\
\hline $\mathrm{IH}-3,18-22$ & 2.75 & 94.85 & 95.10 & 4.90 & 0.49 & 10.1 \\
\hline IH-3, 60-62 & 3.17 & 94.92 & & & & \\
\hline $1 \mathrm{H}-3,118-122$ & 3.75 & 93.81 & 94.36 & 5.64 & 0.40 & 7.0 \\
\hline IH $-4,18-22$ & 4.15 & 95.94 & & & & \\
\hline $1 \mathrm{H}-4,60-62$ & 4.57 & 94.39 & 95.17 & 4.83 & 0.52 & 10.7 \\
\hline $1 \mathrm{H}-4,118-122$ & 5.15 & 96.15 & & & & \\
\hline IH $-5,18-22$ & 5.54 & 95.91 & 96.03 & 3.97 & 0.38 & 9.7 \\
\hline $2 \mathrm{H}-\mathrm{I} .18-22$ & 6.68 & 95.80 & & & & \\
\hline $2 \mathrm{H}-1,60-64$ & 7.10 & 95.74 & 95.77 & 4.23 & 0.36 & 8.5 \\
\hline $2 \mathrm{H}-1,116-118$ & 7.66 & 96.15 & & & & \\
\hline $2 \mathrm{H}-2,18-22$ & 8.10 & 95.96 & 96.06 & 3.94 & 0.24 & 6.0 \\
\hline $2 \mathrm{H}-2,60-64$ & 8.52 & 95.55 & & & & \\
\hline $2 \mathrm{H}-2,116-118$ & 9.08 & 96.66 & 96.11 & 3.89 & 0.25 & 6.5 \\
\hline $2 \mathrm{H}-3,18-22$ & 9.54 & 94.45 & & & & \\
\hline $2 \mathrm{H}-3,60-64$ & 9.96 & 95.46 & 94.96 & 5.04 & 0.24 & 4.7 \\
\hline $2 \mathrm{H}-3,116-118$ & 10.52 & 95.24 & & & & \\
\hline $2 \mathrm{H}-4,18-22$ & 11.04 & 94.98 & 95.11 & 4.89 & 0.25 & 5.1 \\
\hline $2 \mathrm{H}-4,60-64$ & 11.46 & 94.98 & & & & \\
\hline $2 \mathrm{H}-4,116-118$ & 12.02 & 94.66 & 94.82 & 5.18 & 0.18 & 3.5 \\
\hline $2 \mathrm{H}-5,18-22$ & 12.54 & 95.41 & & & & \\
\hline $2 \mathrm{H}-5,60-64$ & 12.96 & 96.91 & 96.16 & 3.84 & 0.20 & 5.3 \\
\hline $2 \mathrm{H}-5,116-118$ & 13.52 & 94.70 & & & & \\
\hline $2 \mathrm{H}-6,18-22$ & 13.96 & 94.71 & 94.71 & 5.29 & 0.23 & 4.4 \\
\hline $3 \mathrm{H}-\mathrm{I}, 18-20$ & 16.18 & 96.43 & & & & \\
\hline $3 \mathrm{H}-1,60-62$ & 16.60 & 95.67 & 96.05 & 3.95 & 0.22 & 5.7 \\
\hline $3 \mathrm{H}-2,18-20$ & 17.24 & 96.64 & & & & \\
\hline $3 \mathrm{H}-2,60-62$ & 17.66 & 95.96 & 96.30 & 3.70 & 0.17 & 4.5 \\
\hline $3 \mathrm{H}-2,116-118$ & 18.22 & 96.92 & & & & \\
\hline $3 \mathrm{H}-3,18-20$ & 18.74 & 96.98 & 96.95 & 3.05 & 0.20 & 6.5 \\
\hline $3 \mathrm{H}-3,60-62$ & 19.16 & 96.17 & & & & \\
\hline $3 \mathrm{H}-3,116-118$ & 19.72 & 93.16 & 94.67 & 5.33 & 0.21 & 3.9 \\
\hline $3 \mathrm{H}-4,18-20$ & 20.24 & 94.80 & & & & \\
\hline $3 \mathrm{H}-4,60-62$ & 20.66 & 96.43 & 95.62 & 4.38 & 0.20 & 4.6 \\
\hline $3 \mathrm{H}-4,116-118$ & 21.22 & 96.20 & & & & \\
\hline $3 \mathrm{H}-5,18-20$ & 21.74 & 96.15 & 96.18 & 3.82 & 0.20 & 5.2 \\
\hline $3 \mathrm{H}-5,60-62$ & 22.16 & 96.35 & & & & \\
\hline $3 \mathrm{H}-5,116-118$ & 22.72 & 95.04 & 95.69 & 4.31 & 0.23 & 5.3 \\
\hline $3 \mathrm{H}-6,18-20$ & 23.24 & 96.24 & & & & \\
\hline $3 \mathrm{H}-6,60-62$ & 23.66 & 96.99 & 96.61 & 3.39 & 0.15 & 4.4 \\
\hline $3 \mathrm{H}-6,116-118$ & 24.22 & 96.32 & & & & \\
\hline $3 \mathrm{H}-7,18-20$ & 24.74 & 96.65 & 96.49 & 3.51 & 0.21 & 5.8 \\
\hline $3 \mathrm{H}-0,18-20$ & 25.33 & 97.09 & & & & \\
\hline $4 \mathrm{H}-1,18-20$ & 25.68 & 97.27 & 97.18 & 2.82 & 0.17 & 6.1 \\
\hline $4 \mathrm{H}-1,60-62$ & 26.10 & 97.04 & & & & \\
\hline $4 \mathrm{H}-1,116-118$ & 26.66 & 96.35 & 96.69 & 3.31 & 0.13 & 4.0 \\
\hline $4 \mathrm{H}-2,18-20$ & 26.87 & 95.98 & & & & \\
\hline
\end{tabular}

\begin{tabular}{|c|c|c|c|c|c|c|}
\hline $\begin{array}{l}\text { Core, section, } \\
\text { interval }(\mathrm{cm})\end{array}$ & $\begin{array}{l}\text { Depth } \\
\text { (mbsf) }\end{array}$ & $\begin{array}{l}\mathrm{CaCO}_{3} \\
(\mathrm{wt} \%)\end{array}$ & $\begin{array}{l}\mathrm{CaCO}_{3} \\
\text { (ave.) } \\
\text { (wt\%) }\end{array}$ & $\begin{array}{c}\text { Noncarbonate } \\
\text { (ave.) } \\
\text { (wt\%) }\end{array}$ & $\begin{array}{l}\text { Eolian } \\
\text { (ave.) } \\
\text { (wt\%) }\end{array}$ & $\begin{array}{l}\text { \%Eolian } \\
\text { carb-free }\end{array}$ \\
\hline $4 \mathrm{H}-2,116-118$ & 27.85 & 96.50 & 96.24 & 3.76 & 0.09 & 2.5 \\
\hline $4 \mathrm{H}-3,18-20$ & 28.34 & 95.25 & & & & \\
\hline $4 \mathrm{H}-3,60-62$ & 28.76 & 96.74 & 95.99 & 4.01 & 0.09 & 2.2 \\
\hline $4 \mathrm{H}-3,116-118$ & 29.32 & 93.62 & & & & \\
\hline $4 \mathrm{H}-4,18-20$ & 29.66 & 97.76 & 95.69 & 4.31 & 0.13 & 2.9 \\
\hline $4 \mathrm{H}-4,60-62$ & 30.08 & 94.58 & & & & \\
\hline $4 \mathrm{H}-4,116-118$ & 30.64 & 96.83 & 95.71 & 4.29 & 0.10 & 2.2 \\
\hline $4 \mathrm{H}-5,18-20$ & 31.16 & 95.91 & & & & \\
\hline $4 \mathrm{H}-5,60-62$ & 31.58 & 93.77 & 94.84 & 5.16 & 0.08 & 1.6 \\
\hline $4 \mathrm{H}-5,116-118$ & 32.14 & 94.62 & & & & \\
\hline $4 \mathrm{H}-6,18-20$ & 32.66 & 94.33 & 94.48 & 5.52 & 0.09 & 1.6 \\
\hline $5 \mathrm{H}-1,18-20$ & 35.18 & 92.46 & & & & \\
\hline $5 \mathrm{H}-1,61-63$ & 35.61 & 89.66 & 91.06 & 8.94 & 0.09 & 1.0 \\
\hline $5 \mathrm{H}-2,18-20$ & 35.81 & 93.01 & & & & \\
\hline $5 \mathrm{H}-2,61-63$ & 36.24 & 88.77 & 90.89 & 9.11 & 0.07 & 0.8 \\
\hline $5 \mathrm{H}-2,116-118$ & 36.79 & 92.07 & & & & \\
\hline $5 \mathrm{H}-3,18-20$ & 37.08 & 92.28 & 92.18 & 7.82 & 0.07 & 0.8 \\
\hline $5 \mathrm{H}-3,66-68$ & 37.56 & 91.30 & & & & \\
\hline $5 \mathrm{H}-3,116-118$ & 38.06 & 92.14 & 91.72 & 8.28 & 0.05 & 0.6 \\
\hline $5 \mathrm{H}-4,18-20$ & 38.39 & 93.98 & & & & \\
\hline $5 \mathrm{H}-4,61-63$ & 38.82 & 92.90 & 93.44 & 6.56 & 0.04 & 0.6 \\
\hline $5 \mathrm{H}-4,116-118$ & 39.37 & 94.84 & & & & \\
\hline $5 \mathrm{H}-5,18-20$ & 39.79 & 91.29 & 93.07 & 6.93 & 0.02 & 0.3 \\
\hline $5 \mathrm{H}-5,61-63$ & 40.22 & 95.15 & & & & \\
\hline $5 \mathrm{H}-5,116-118$ & 40.77 & 91.99 & 93.57 & 6.43 & 0.02 & 0.4 \\
\hline $5 \mathrm{H}-6,18-20$ & 41.18 & 93.96 & & & & \\
\hline $5 \mathrm{H}-6,61-63$ & 41.61 & 93.09 & 93.52 & 6.48 & 0.03 & 0.4 \\
\hline $6 \mathrm{H}-1,18-20$ & 44.68 & 94.35 & & & & \\
\hline $6 \mathrm{H}-1,60-62$ & 45.10 & 94.11 & 94.23 & 5.77 & 0.34 & 5.8 \\
\hline $6 \mathrm{H}-1,116-118$ & 45.66 & 95.97 & & & & \\
\hline $6 \mathrm{H}-2,18-20$ & 45.92 & 92.26 & 94.12 & 5.88 & 0.29 & 5.0 \\
\hline $6 \mathrm{H}-2,60-62$ & 46.34 & 93.66 & & & & \\
\hline $6 \mathrm{H}-2,116-118$ & 46.90 & 93.81 & 93.74 & 6.26 & 0.37 & 5.9 \\
\hline $6 \mathrm{H}-3,18-20$ & 47.33 & 93.93 & & & & \\
\hline $6 \mathrm{H}-3,60-62$ & 47.75 & 92.23 & 93.08 & 6.92 & 0.07 & 1.1 \\
\hline $6 \mathrm{H}-3,116-118$ & 48.31 & 94.40 & & & & \\
\hline $6 \mathrm{H}-4,18-20$ & 48.71 & 94.04 & 94.22 & 5.78 & 0.07 & 1.2 \\
\hline $6 \mathrm{H}-4,60-62$ & 49.13 & 94.35 & & & & \\
\hline $6 \mathrm{H}-4,116-118$ & 49.69 & 94.26 & 94.31 & 5.69 & 0.12 & 2.1 \\
\hline $6 \mathrm{H}-5,18-20$ & 50.07 & 94.96 & & & & \\
\hline $6 \mathrm{H}-5,60-62$ & 50.49 & 94.05 & 94.51 & 5.49 & 0.12 & 2.2 \\
\hline $6 \mathrm{H}-5,116-118$ & 51.05 & 91.13 & & & & \\
\hline $6 \mathrm{H}-6,18-20$ & 51.45 & 94.35 & 92.74 & 7.26 & 0.10 & 1.4 \\
\hline
\end{tabular}

Notes: $\mathrm{CaCO}_{3}=$ weight percentage of calcium carbonate in bulk sample, Noncarbonate $=$ percentage of noncarbonate in bulk sample (calculated as $100 \%-\mathrm{wt} \% \mathrm{CaCO}_{3}$ ), Eolian $=$ weight percentage of the extracted "eolian," or fine-grained $(<63 \mu \mathrm{m})$ lithogenic fraction of the bulk sediment, and \%Eolian (carb-free) = weight percentage of the extracted "eolian" fraction relative to the noncarbonate weight percentage, rather than to the total bulk sample. Average values are given where two successive samples were used to conduct the chemical extraction procedure.

Table 8. Age models for Site 873.

\begin{tabular}{rrrrrl}
\hline $\begin{array}{c}\text { Depth } \\
\text { top } \\
\text { (mbsf) }\end{array}$ & $\begin{array}{c}\text { Depth } \\
\text { bottom } \\
\text { (mbsf) }\end{array}$ & $\begin{array}{r}\text { Depth } \\
\text { middle } \\
\text { (mbsf) }\end{array}$ & $\begin{array}{c}\text { Age } \\
\text { (Ma) }\end{array}$ & Code & \multicolumn{1}{c}{ Datum } \\
\hline 0.00 & 0.05 & 0.03 & 0.470 & C1 & LAD P. lacunosa \\
0.00 & 0.05 & 0.03 & 1.370 & C2 & LAD H. sellit** \\
3.07 & 4.02 & 3.55 & 1.450 & C3 & LAD C. macintyrei \\
4.45 & 4.95 & 4.70 & 1.900 & C4 & LAD D. brouweri \\
4.45 & 4.95 & 4.70 & 1.900 & C5 & LAD D. triradiatus \\
5.38 & 6.59 & 5.99 & 2.200 & C6 & LAD D. aymmetricus \\
7.12 & 8.57 & 7.84 & 2.400 & C7 & LAD D. pentaradiatus \\
9.88 & 11.38 & 10.63 & 2.600 & C8 & LAD D. tamalis \\
14.53 & 16.50 & 15.51 & 3.470 & C10 & LAD $S$. abies + S. neoabies \\
16.50 & 17.57 & 17.03 & 3.500 & C11 & LAD $R$. pseudoumbilica \\
20.56 & 22.06 & 21.31 & 5.000 & C12 & LAD T. rugosus ** \\
25.48 & 25.55 & 25.51 & 11.600 & C13 & LAD C. floridanus** \\
42.48 & 41.92 & 42.20 & 14.400 & C14 & LAD . heteromorphus** \\
51.82 & 54.01 & 52.92 & 17.100 & C15 & FAD $S$. heteromorphus ** \\
\hline
\end{tabular}

Notes: Age models were generated using the midpoint depth (mbsf) for each datum. Double asterisks $(* *)$ indicate the datums that were used to generate the "simplified" version of the age model. FAD $=$ first appearance datum, and $\mathrm{LAD}=$ last appearance datum. Codes are taken from Premoli Silva, Haggerty, Rack, et al. (1993). C = nannofossil datum, and $\mathrm{F}=$ foraminifer datum. 
Table 9. Linear sedimentation and mass accumulation rates for Hole 873B, calculated using the comprehensive and simplified age models.

\begin{tabular}{|c|c|c|c|c|c|c|c|c|c|}
\hline $\begin{array}{l}\text { Core, section, } \\
\text { interval }(\mathrm{cm})\end{array}$ & $\begin{array}{l}\text { Depth } \\
\text { (mbsf) }\end{array}$ & $\begin{array}{l}\text { Age } \\
\text { (Ma) }\end{array}$ & $\begin{array}{c}\text { LSR } \\
(\mathrm{cm} / \mathrm{k} . \mathrm{y} .)\end{array}$ & $\underset{\left(\mathrm{g} / \mathrm{cm}^{3}\right)}{\text { DBD }}$ & MAR & $\begin{array}{l}\text { MAR } \\
\text { (ave.) }\end{array}$ & CAR & $\begin{array}{l}\text { CAR } \\
\text { (ave.) }\end{array}$ & $\begin{array}{l}\text { NCAR } \\
\text { (ave.) }\end{array}$ \\
\hline $\begin{array}{l}\text { Comprehensive n } \\
\text { 144-873B- }\end{array}$ & del: & & & & & & & & \\
\hline $1 \mathrm{H}-1,60$ & 0.60 & 1.383 & 4.400 & 0.97 & 4.268 & & 3.897 & & \\
\hline $1 \mathrm{H}-1,117$ & 1.17 & 1.396 & 4.400 & 0.92 & 4.048 & 4.158 & 3.803 & 3.851 & 0.055 \\
\hline $1 \mathrm{H}-2,18$ & 1.37 & 1.401 & 4.400 & 0.86 & 3.784 & & 3.512 & & \\
\hline IH- 2,60 & 1.79 & 1.410 & 4.400 & 0.86 & 3.784 & 3.784 & 3.554 & 3.533 & 0.251 \\
\hline $1 \mathrm{H}-2,118$ & 2.37 & 1.423 & 4.400 & 0.92 & 4.048 & & 3.859 & & \\
\hline $1 \mathrm{H}-3,18$ & 2.75 & 1.432 & 4.400 & 0.90 & 3.960 & 4.004 & 3.756 & 3.808 & 0.196 \\
\hline $1 \mathrm{H}-3,60$ & 3.17 & 1.441 & 4.400 & 0.90 & 3.960 & & 3.759 & & \\
\hline $1 \mathrm{H}-3,118$ & 3.75 & 1.528 & 0.256 & 0.92 & 0.236 & 2.098 & 0.221 & 1.980 & 0.118 \\
\hline IH-4, 18 & 4.15 & 1.685 & 0.256 & 1.01 & 0.258 & & 0.248 & & \\
\hline $1 \mathrm{H}-4,60$ & 4.57 & 1.849 & 0.256 & 1.00 & 0.256 & 0.257 & 0.241 & 0.244 & 0.012 \\
\hline $1 \mathrm{H}-4,118$ & 5.15 & 2.005 & 0.430 & 1.02 & 0.439 & & 0.422 & & \\
\hline IH- 5,18 & 5.54 & 2.095 & 0.430 & 1.00 & 0.430 & 0.435 & 0.413 & 0.417 & 0.017 \\
\hline $2 \mathrm{H}-1,18$ & 6.68 & 2.275 & 0.925 & 1.01 & 0.934 & & 0.895 & & \\
\hline $2 \mathrm{H}-1,60$ & 7.10 & 2.320 & 0.925 & 1.00 & 0.925 & 0.930 & 0.886 & 0.890 & 0.039 \\
\hline $2 \mathrm{H}-1,116$ & 7.66 & 2.381 & 0.925 & 0.95 & 0.879 & & 0.845 & & \\
\hline $2 \mathrm{H}-2,18$ & 8.10 & 2.419 & 1.395 & 0.96 & 1.339 & 1.109 & 1.285 & 1.065 & 0.044 \\
\hline $2 \mathrm{H}-2,60$ & 8.52 & 2.449 & 1.395 & 0.93 & 1.298 & & 1.240 & & \\
\hline $2 \mathrm{H}-2,116$ & 9.08 & 2.489 & 1.395 & 0.94 & 1.311 & 1.304 & 1.268 & 1.254 & 0.051 \\
\hline $2 \mathrm{H}-3,18$ & 9.54 & 2.522 & 1.395 & 0.97 & 1.353 & & 1.278 & & \\
\hline $2 \mathrm{H}-3,60$ & 9.96 & 2.552 & 1.395 & 0.91 & 1.270 & 1.311 & 1.212 & 1.245 & 0.066 \\
\hline $2 \mathrm{H}-3,116$ & 10.52 & 2.592 & 1.395 & 0.94 & 1.311 & & 1.249 & & \\
\hline $2 \mathrm{H}-4,18$ & 11.04 & 2.673 & 0.561 & 0.96 & 0.539 & 0.925 & 0.512 & 0.880 & 0.045 \\
\hline $2 \mathrm{H}-4,60$ & 11.46 & 2.748 & 0.561 & 0.92 & 0.516 & & 0.490 & & \\
\hline $2 \mathrm{H}-4,116$ & 12.02 & 2.848 & 0.561 & 0.87 & 0.488 & 0.502 & 0.462 & 0.476 & 0.026 \\
\hline $2 \mathrm{H}-5,18$ & 12.54 & 2.941 & 0.561 & 0.90 & 0.505 & & 0.482 & & \\
\hline $2 \mathrm{H}-5,60$ & 12.96 & 3.015 & 0.561 & 0.90 & 0.505 & 0.505 & 0.489 & 0.485 & 0.019 \\
\hline $2 \mathrm{H}-5,116$ & 13.52 & 3.115 & 0.561 & 0.96 & 0.539 & & 0.510 & & \\
\hline $2 \mathrm{H}-6,18$ & 13.96 & 3.194 & 0.561 & 0.93 & 0.521 & 0.530 & 0.494 & 0.502 & 0.028 \\
\hline $3 \mathrm{H}-1,18$ & 16.18 & 3.483 & 5.067 & 0.91 & 4.611 & & 4.446 & & \\
\hline $3 \mathrm{H}-1,60$ & 16.60 & 3.491 & 5.067 & 0.88 & 4.459 & 4.535 & 4.266 & 4.356 & 0.179 \\
\hline $3 \mathrm{H}-2,18$ & 17.24 & 3.574 & 0.285 & 0.89 & 0.254 & & 0.245 & & \\
\hline $3 \mathrm{H}-2,60$ & 17.66 & 3.721 & 0.285 & 0.86 & 0.245 & 0.250 & 0.235 & 0.240 & 0.009 \\
\hline $3 \mathrm{H}-2,116$ & 18.22 & 3.917 & 0.285 & 0.89 & 0.254 & & 0.246 & & \\
\hline $3 \mathrm{H}-3,18$ & 18.74 & 4.099 & 0.285 & 0.88 & 0.251 & 0.253 & 0.244 & 0.245 & 0.008 \\
\hline $3 \mathrm{H}-3,60$ & 19.16 & 4.247 & 0.285 & 0.90 & 0.257 & & 0.247 & & \\
\hline $3 \mathrm{H}-3,116$ & 19.72 & 4.443 & 0.285 & 0.91 & 0.260 & 0.258 & 0.242 & 0.244 & 0.014 \\
\hline $3 \mathrm{H}-4,18$ & 20.24 & 4.625 & 0.285 & 1.00 & 0.285 & & 0.271 & & \\
\hline $3 \mathrm{H}-4,60$ & 20.66 & 4.772 & 0.285 & 0.98 & 0.280 & 0.283 & 0.270 & 0.270 & 0.012 \\
\hline $3 \mathrm{H}-4,116$ & 21.22 & 4.969 & 0.285 & 1.00 & 0.285 & & 0.274 & & \\
\hline $3 \mathrm{H}-5,18$ & 21.74 & 5.676 & 0.064 & 1.02 & 0.065 & 0.175 & 0.063 & 0.169 & 0.007 \\
\hline $3 \mathrm{H}-5,60$ & 22.16 & 6.336 & 0.064 & 1.05 & 0.067 & & 0.064 & & \\
\hline $3 \mathrm{H}-5,116$ & 22.72 & 7.216 & 0.064 & 0.98 & 0.062 & 0.065 & 0.059 & 0.062 & 0.003 \\
\hline $3 \mathrm{H}-6,18$ & 23.24 & 8.033 & 0.064 & 0.95 & 0.060 & & 0.058 & & \\
\hline $3 \mathrm{H}-6,60$ & 23.66 & 8.693 & 0.064 & 0.98 & 0.062 & 0.061 & 0.060 & 0.059 & 0.002 \\
\hline $3 \mathrm{H}-6,116$ & 24.22 & 9.573 & 0.064 & 1.08 & 0.069 & & 0.066 & & \\
\hline $3 \mathrm{H}-7,18$ & 24.74 & 10.390 & 0.064 & 1.06 & 0.067 & 0.068 & 0.065 & 0.066 & 0.002 \\
\hline $3 \mathrm{H}-0,18$ & 25.33 & 11.317 & 0.064 & 0.97 & 0.062 & & 0.060 & & \\
\hline $4 \mathrm{H}-1,18$ & 25.68 & 11.628 & 0.596 & 0.98 & 0.584 & 0.323 & 0.568 & 0.314 & 0.009 \\
\hline $4 \mathrm{H}-1,60$ & 26.10 & 11.699 & 0.596 & 0.98 & 0.584 & & 0.567 & & \\
\hline $4 \mathrm{H}-1,116$ & 26.66 & 11.793 & 0.596 & 0.89 & 0.531 & 0.557 & 0.511 & 0.539 & 0.018 \\
\hline $4 \mathrm{H}-2,18$ & 26.87 & 11.828 & 0.595 & 0.95 & 0.566 & & 0.543 & & \\
\hline $4 \mathrm{H}-2,116$ & 27.85 & 11.993 & 0.596 & 0.87 & 0.519 & 0.542 & 0.500 & 0.522 & 0.020 \\
\hline $4 \mathrm{H}-3,18$ & 28.34 & 12.075 & 0.596 & 0.82 & 0.489 & & 0.466 & & \\
\hline $4 \mathrm{H}-3,60$ & 28.76 & 12.145 & 0.596 & 0.88 & 0.524 & 0.507 & 0.507 & 0.486 & 0.020 \\
\hline $4 \mathrm{H}-3,116$ & 29.32 & 12.239 & 0.596 & 0.87 & 0.518 & & 0.485 & & \\
\hline $4 \mathrm{H}-4,18$ & 29.66 & 12.296 & 0.596 & 0.84 & 0.501 & 0.510 & 0.490 & 0.488 & 0.022 \\
\hline $4 \mathrm{H}-4,60$ & 30.08 & 12.367 & 0.596 & 0.91 & 0.542 & & 0.513 & & \\
\hline $4 \mathrm{H}-4,116$ & 30.64 & 12.461 & 0.596 & 0.84 & 0.501 & 0.522 & 0.485 & 0.499 & 0.022 \\
\hline $4 \mathrm{H}-5,18$ & 31.16 & 12.548 & 0.596 & 0.80 & 0.477 & & 0.457 & & \\
\hline $4 \mathrm{H}-5,60$ & 31.58 & 12.618 & 0.596 & 0.83 & 0.495 & 0.486 & 0.464 & 0.461 & 0.025 \\
\hline $4 \mathrm{H}-5,116$ & 32.14 & 12.712 & 0.596 & 0.82 & 0.489 & & 0.462 & & \\
\hline $4 \mathrm{H}-6,18$ & 32.66 & 12.800 & 0.596 & 0.74 & 0.441 & 0.465 & 0.416 & 0.439 & 0.026 \\
\hline $5 \mathrm{H}-1,18$ & 35.18 & 13.222 & 0.596 & 0.67 & 0.399 & & 0.369 & & \\
\hline $5 \mathrm{H}-1,61$ & 35.61 & 13.294 & 0.596 & 0.66 & 0.394 & 0.396 & 0.353 & 0.361 & 0.035 \\
\hline $5 \mathrm{H}-2,18$ & 35.81 & 13.328 & 0.596 & 0.61 & 0.364 & & 0.338 & & \\
\hline $5 \mathrm{H}-2,61$ & 36.24 & 13.400 & 0.596 & 0.63 & 0.376 & 0.370 & 0.334 & 0.336 & 0.034 \\
\hline $5 \mathrm{H}-2,116$ & 36.79 & 13.492 & 0.596 & 0.63 & 0.375 & & 0.346 & & \\
\hline $5 \mathrm{H}-3,18$ & 37.08 & 13.541 & 0.596 & 0.65 & 0.387 & 0.381 & 0.357 & 0.352 & 0.030 \\
\hline $5 \mathrm{H}-3,66$ & 37.56 & 13.622 & 0.596 & 0.76 & 0.453 & & 0.413 & & \\
\hline $5 \mathrm{H}-3,116$ & 38.06 & 13.705 & 0.596 & 0.66 & 0.393 & 0.423 & 0.362 & 0.388 & 0.035 \\
\hline $5 \mathrm{H}-4,18$ & 38.39 & 13.761 & 0.596 & 0.63 & 0.375 & & 0.353 & & \\
\hline $5 \mathrm{H}-4,6 \mathrm{I}$ & 38.82 & 13.833 & 0.596 & 0.80 & 0.476 & 0.426 & 0.443 & 0.398 & 0.028 \\
\hline $5 \mathrm{H}-4,116$ & 39.37 & 13.925 & 0.596 & 0.82 & 0.489 & & 0.464 & & \\
\hline $5 \mathrm{H}-5,18$ & 39.79 & 13.996 & 0.596 & 0.76 & 0.453 & 0.471 & 0.413 & 0.438 & 0.033 \\
\hline $5 \mathrm{H}-5,61$ & 40.22 & 14.068 & 0.596 & 0.81 & 0.483 & & 0.460 & & \\
\hline $5 \mathrm{H}-5,116$ & 40.77 & 14.160 & 0.596 & 0.79 & 0.471 & 0.477 & 0.433 & 0.446 & 0.031 \\
\hline $5 \mathrm{H}-6,18$ & 41.18 & 14.229 & 0.596 & 0.79 & 0.471 & & 0.442 & & \\
\hline $5 \mathrm{H}-6,61$ & 41.61 & 14.301 & 0.596 & 0.75 & 0.447 & 0.459 & 0.416 & 0.429 & 0.030 \\
\hline $6 \mathrm{H}-1,18$ & 44.68 & 15.025 & 0.397 & 0.71 & 0.282 & & 0.266 & & \\
\hline $6 \mathrm{H}-1,60$ & 45.10 & 15.130 & 0.397 & 0.78 & 0.310 & 0.296 & 0.291 & 0.279 & 0.017 \\
\hline $6 \mathrm{H}-1,116$ & 45.66 & 15.271 & 0.397 & 0.61 & 0.242 & & 0.232 & & \\
\hline $6 \mathrm{H}-2,18$ & 45.92 & 15.337 & 0.397 & 0.60 & 0.238 & 0.240 & 0.220 & 0.226 & 0.014 \\
\hline $6 \mathrm{H}-2,60$ & 46.34 & 15.443 & 0.397 & 0.69 & 0.274 & & 0.257 & & \\
\hline $6 \mathrm{H}-2,116$ & 46.90 & 15.584 & 0.397 & 0.68 & 0.270 & 0.272 & 0.253 & 0.255 & 0.017 \\
\hline $6 \mathrm{H}-3,18$ & 47.33 & 15.692 & 0.397 & 0.69 & 0.274 & & 0.257 & & \\
\hline $\begin{array}{l}6 \mathrm{H}-3,60 \\
6 \mathrm{H}-3,16\end{array}$ & $\begin{array}{l}47.75 \\
48.31\end{array}$ & $\begin{array}{l}15.798 \\
15.939\end{array}$ & $\begin{array}{l}0.397 \\
0.397\end{array}$ & $\begin{array}{l}0.66 \\
0.73\end{array}$ & 0.262 & 0.268 & 0.242 & 0.249 & 0.019 \\
\hline & 48.31 & 15.939 & 0.397 & 0.73 & 0.290 & & 0.274 & & \\
\hline
\end{tabular}


Table 9 (continued).

\begin{tabular}{|c|c|c|c|c|c|c|c|c|c|c|}
\hline $\begin{array}{l}\text { Core, section, } \\
\text { interval }(\mathrm{cm})\end{array}$ & $\begin{array}{l}\text { Depth } \\
\text { (mbsf) }\end{array}$ & $\begin{array}{l}\text { Age } \\
(\mathrm{Ma})\end{array}$ & $\begin{array}{c}\text { LSR } \\
(\mathrm{cm} / \mathrm{k} . \mathrm{y} .)\end{array}$ & $\begin{array}{c}\text { DBD } \\
\left(\mathrm{g} / \mathrm{cm}^{3}\right)\end{array}$ & MAR & $\begin{array}{l}\text { MAR } \\
\text { (ave.) }\end{array}$ & CAR & $\begin{array}{l}\text { CAR } \\
\text { (ave.) }\end{array}$ & $\begin{array}{l}\text { NCAR } \\
\text { (ave.) }\end{array}$ & $\begin{array}{l}\text { EAR } \\
\text { (ave.) }\end{array}$ \\
\hline $6 \mathrm{H}-4,18$ & 48.71 & 16.040 & 0.397 & 0.76 & 0.302 & 0.296 & 0.284 & 0.279 & 0.017 & 0.0002 \\
\hline $6 \mathrm{H}-4,60$ & 49.13 & 16.145 & 0.397 & 0.77 & 0.306 & & 0.288 & & & \\
\hline $6 \mathrm{H}-4,116$ & 49.69 & 16.287 & 0.397 & 0.77 & 0.306 & 0.306 & 0.288 & 0.288 & 0.017 & 0.0004 \\
\hline $6 \mathrm{H}-5,18$ & 50.07 & 16.382 & 0.397 & 0.62 & 0.246 & & 0.234 & & & \\
\hline $6 \mathrm{H}-5,60$ & 50,49 & 16.488 & 0.397 & 0.62 & 0.246 & 0.246 & 0.231 & 0.233 & 0.014 & 0.0003 \\
\hline $6 \mathrm{H}-5,116$ & 51.05 & 16.629 & 0.397 & 0.72 & 0.286 & & 0.261 & & & \\
\hline $6 \mathrm{H}-6,18$ & 51.45 & 16.730 & 0.397 & 0.64 & 0.254 & 0.270 & 0.240 & 0.250 & 0.020 & 0.0003 \\
\hline $\begin{array}{l}\text { Simplified age m } \\
\text { 144-873B- }\end{array}$ & & & & & & & & & & \\
\hline $1 \mathrm{H}-1,60$ & 0.60 & 1.467 & 0.586 & 0.97 & 0.568 & & 0.519 & & & \\
\hline $1 \mathrm{H}-1,117$ & 1.17 & 1.564 & 0.586 & 0.92 & 0.539 & 0.554 & 0.506 & 0.513 & 0.021 & 0.0013 \\
\hline IH-2, 18 & 1.37 & 1.599 & 0.587 & 0.86 & 0.504 & & 0.468 & & & \\
\hline IH-2, 60 & 1.79 & 1.670 & 0.587 & 0.86 & 0.504 & 0.504 & 0.474 & 0.471 & 0.033 & 0.0017 \\
\hline $1 \mathrm{H}-2,118$ & 2.37 & 1.769 & 0.586 & 0.92 & 0.539 & & 0.514 & & & \\
\hline IH-3, 18 & 2.75 & 1.834 & 0.586 & 0.90 & 0.528 & 0.533 & 0.501 & 0.507 & 0.026 & 0.0026 \\
\hline $1 \mathrm{H}-3,60$ & 3.17 & 1.906 & 0.587 & 0.90 & 0.528 & & 0.501 & & & \\
\hline IH-3, 118 & 3.75 & 2.005 & 0.586 & 0.92 & 0.539 & 0.533 & 0.506 & 0.503 & 0.030 & 0.0021 \\
\hline IH-4, 18 & 4.15 & 2.073 & 0.587 & 1.01 & 0.592 & & 0.568 & & & \\
\hline $\mathrm{IH}-4,60$ & 4.57 & 2.144 & 0.587 & 1.00 & 0.587 & 0.589 & 0.554 & 0.561 & 0.029 & 0.0030 \\
\hline $\mathrm{IH}-4,118$ & 5.15 & 2.243 & 0.586 & 1.02 & 0.598 & & 0.575 & & & \\
\hline IH- 5,18 & 5.54 & 2.310 & 0.586 & 1.00 & 0.586 & 0.592 & 0.562 & 0.569 & 0.024 & 0.0023 \\
\hline $2 \mathrm{H}-1,18$ & 6.68 & 2.504 & 0.586 & 1.01 & 0.592 & & 0.567 & & & \\
\hline $2 \mathrm{H}-1,60$ & 7.10 & 2.576 & 0.587 & 1.00 & 0.587 & 0.589 & 0.562 & 0.564 & 0.025 & 0.0021 \\
\hline $2 \mathrm{H}-1,116$ & 7.66 & 2.671 & 0.586 & 0.95 & 0.557 & & 0.536 & & & \\
\hline $2 \mathrm{H}-2,18$ & 8.10 & 2.747 & 0.586 & 0.96 & 0.562 & 0.560 & 0.540 & 0.538 & 0.022 & 0.0013 \\
\hline $2 \mathrm{H}-2,60$ & 8.52 & 2.818 & 0.587 & 0.93 & 0.546 & & 0.521 & & & \\
\hline $2 \mathrm{H}-2,116$ & 9.08 & 2.914 & 0.586 & 0.94 & 0.551 & 0.548 & 0.532 & 0.527 & 0.021 & 0.0014 \\
\hline $2 \mathrm{H}-3,18$ & 9.54 & 2.992 & 0.587 & 0.97 & 0.569 & & 0.538 & & & \\
\hline $2 \mathrm{H}-3,60$ & 9.96 & 3.064 & 0.586 & 0.91 & 0.533 & 0.551 & 0.509 & 0.523 & 0.028 & 0.0013 \\
\hline $2 \mathrm{H}-3,116$ & 10.52 & 3.159 & 0.586 & 0.94 & 0.551 & & 0.525 & & & \\
\hline $2 \mathrm{H}-4,18$ & 11.04 & 3.248 & 0.586 & 0.96 & 0.563 & 0.557 & 0.535 & 0.530 & 0.027 & 0.0014 \\
\hline $2 \mathrm{H}-4,60$ & 11.46 & 3.320 & 0.586 & 0.92 & 0.539 & & 0.512 & & & \\
\hline $2 \mathrm{H}-4,116$ & 12.02 & 3.415 & 0.586 & 0.87 & 0.510 & 0.525 & 0.483 & 0.497 & 0.027 & 0.0009 \\
\hline $2 \mathrm{H}-5,18$ & 12.54 & 3.504 & 0.586 & 0.90 & 0.528 & & 0.503 & & & \\
\hline $2 \mathrm{H}-5,60$ & 12.96 & 3.576 & 0.587 & 0.90 & 0.528 & 0.528 & 0.512 & 0.508 & 0.020 & 0.0011 \\
\hline $2 \mathrm{H}-5,116$ & 13.52 & 3.671 & 0.586 & 0.96 & 0.562 & & 0.533 & & & \\
\hline $2 \mathrm{H}-6,18$ & 13.96 & 3.746 & 0.587 & 0.93 & 0.546 & 0.554 & 0.517 & 0.525 & 0.029 & 0.0013 \\
\hline $3 \mathrm{H}-1,18$ & 16.18 & 4.125 & 0.586 & 0.91 & 0.533 & & 0.514 & & & \\
\hline $3 \mathrm{H}-1,60$ & 16.60 & 4.197 & 0.586 & 0.88 & 0.515 & 0.524 & 0.493 & 0.504 & $0.02 \mathrm{I}$ & 0.0012 \\
\hline $3 \mathrm{H}-2,18$ & 17.24 & 4.306 & 0.587 & 0.89 & 0.522 & & 0.505 & & & \\
\hline $3 \mathrm{H}-2,60$ & 17.66 & 4.377 & 0.586 & 0.86 & 0.504 & 0.513 & 0.483 & 0.494 & 0.019 & 0.0008 \\
\hline $3 \mathrm{H}-2,116$ & 18.22 & 4.473 & 0.586 & 0.89 & 0.522 & & 0.506 & & & \\
\hline $3 \mathrm{H}-3,18$ & 18.74 & 4.562 & 0.586 & 0.88 & 0.516 & 0.519 & 0.500 & 0.503 & 0.016 & 0.0010 \\
\hline $3 \mathrm{H}-3,60$ & 19.16 & 4.633 & 0.587 & 0.90 & 0.528 & & 0.508 & & & \\
\hline $3 \mathrm{H}-3,116$ & 19.72 & 4.729 & 0.586 & 0.91 & 0.533 & 0.530 & 0.497 & 0.502 & 0.028 & 0.0011 \\
\hline $3 \mathrm{H}-4,18$ & 20.24 & 4.818 & 0.586 & 1.00 & 0.586 & & 0.556 & & & \\
\hline $3 \mathrm{H}-4,60$ & 20.66 & 4.889 & 0.587 & 0.98 & 0.575 & 0.581 & 0.554 & 0.555 & 0.025 & 0.0012 \\
\hline $3 \mathrm{H}-4,116$ & 21.22 & 4.985 & 0.586 & 1.00 & 0.586 & & 0.564 & & & \\
\hline $3 \mathrm{H}-5,18$ & 21.74 & 5.676 & 0.064 & 1.02 & 0.065 & 0.326 & 0.063 & 0.314 & 0.003 & 0.0001 \\
\hline $3 \mathrm{H}-5,60$ & 22.16 & 6.336 & 0.064 & 1.05 & 0.067 & & 0.064 & & & \\
\hline $3 \mathrm{H}-5,116$ & 22.72 & 7.216 & 0.064 & 0.98 & 0.062 & 0.065 & 0.059 & 0.062 & 0.003 & 0.0001 \\
\hline $3 \mathrm{H}-6,18$ & 23.24 & 8.033 & 0.064 & 0.95 & 0.060 & & 0.058 & & & \\
\hline $3 \mathrm{H}-6,60$ & 23.66 & 8.693 & 0.064 & 0.98 & 0.062 & 0.061 & 0.060 & 0.059 & 0.002 & 0.0001 \\
\hline $3 \mathrm{H}-6,116$ & 24.22 & 9.573 & 0.064 & 1.08 & 0.069 & & 0.066 & & & \\
\hline $3 \mathrm{H}-7.18$ & 24.74 & 10.390 & 0.064 & 1.06 & 0.067 & 0.068 & 0.065 & 0.066 & 0.002 & 0.0001 \\
\hline $3 \mathrm{H}-0,18$ & 25.33 & 11.317 & 0.064 & 0.97 & 0.062 & & 0.060 & & & \\
\hline $4 \mathrm{H}-1,18$ & 25.68 & 11.628 & 0.596 & 0.98 & 0.584 & 0.086 & 0.568 & 0.315 & 0.016 & 0.0001 \\
\hline $4 \mathrm{H}-1,60$ & 26.10 & 11.699 & 0.596 & 0.98 & 0.584 & & 0.567 & & & \\
\hline $4 \mathrm{H}-1,116$ & 26.66 & 11.793 & 0.596 & 0.89 & 0.531 & 0.557 & 0.511 & 0.539 & 0.018 & 0.0007 \\
\hline $4 \mathrm{H}-2,18$ & 26.87 & 11.828 & 0.595 & 0.95 & 0.565 & & 0.542 & & & \\
\hline $4 \mathrm{H}-2,116$ & 27.85 & 11.993 & 0.596 & 0.87 & 0.519 & 0.542 & 0.500 & 0.522 & 0.020 & 0.0005 \\
\hline $4 \mathrm{H}-3,18$ & 28.34 & 12.075 & 0.596 & 0.82 & 0.489 & & 0.466 & & & \\
\hline $4 \mathrm{H}-3,60$ & 28.76 & 12.145 & 0.597 & 0.88 & 0.525 & 0.507 & 0.508 & 0.487 & 0.020 & 0.0005 \\
\hline $4 \mathrm{H}-3,116$ & 29.32 & 12.239 & 0.596 & 0.87 & 0.518 & & 0.485 & & & \\
\hline $4 \mathrm{H}-4,18$ & 29.66 & 12.296 & 0.596 & 0.84 & 0.501 & 0.510 & 0.490 & 0.488 & 0.022 & 0.0006 \\
\hline $4 \mathrm{H}-4,60$ & 30.08 & 12.367 & 0.596 & 0.91 & 0.542 & & 0.513 & & & \\
\hline $4 \mathrm{H}-4,116$ & 30.64 & 12.461 & 0.596 & 0.84 & 0.501 & 0.522 & 0.485 & 0.499 & 0.022 & 0.0005 \\
\hline $4 \mathrm{H}-5,18$ & 31.16 & 12.548 & 0.596 & 0.80 & 0.477 & & 0.457 & & & \\
\hline $4 \mathrm{H}-5,60$ & 31.58 & 12.618 & 0.597 & 0.83 & 0.495 & 0.486 & 0.464 & 0.461 & 0.025 & 0.0004 \\
\hline $4 \mathrm{H}-5,116$ & 32.14 & 12.712 & 0.596 & 0.82 & 0.489 & & 0.462 & & & \\
\hline $4 \mathrm{H}-6,18$ & 32.66 & 12.800 & 0.596 & 0.74 & 0.441 & 0.465 & 0.416 & 0.439 & 0.026 & 0.0004 \\
\hline $5 \mathrm{H}-1,18$ & 35.18 & 13.222 & 0.596 & 0.67 & 0.399 & & 0.369 & & & \\
\hline $5 \mathrm{H}-1,61$ & 35.61 & 13.294 & 0.596 & 0.66 & 0.394 & 0.396 & 0.353 & 0.361 & 0.035 & 0.0004 \\
\hline $5 \mathrm{H}-2,18$ & 35.81 & 13.328 & 0.595 & 0.61 & 0.363 & & 0.338 & & & \\
\hline $5 \mathrm{H}-2,61$ & 36.24 & 13.400 & 0.596 & 0.63 & 0.376 & 0.369 & 0.334 & 0.336 & 0.034 & 0.0003 \\
\hline $5 \mathrm{H}-2,116$ & 36.79 & 13.492 & 0.596 & 0.63 & 0.375 & & 0.346 & & & \\
\hline $5 \mathrm{H}-3,18$ & 37.08 & 13.541 & 0.597 & 0.65 & 0.388 & 0.382 & 0.358 & 0.352 & 0.030 & 0.0002 \\
\hline $5 \mathrm{H}-3,66$ & 37.56 & 13.622 & 0.596 & 0.76 & 0.453 & & 0.413 & & & \\
\hline $5 \mathrm{H}-3,116$ & 38.06 & 13.705 & 0.596 & 0.66 & 0.393 & 0.423 & 0.362 & 0.388 & 0.035 & 0.0002 \\
\hline SH-4. 18 & 38.39 & 13.761 & 0.597 & 0.63 & 0.376 & & 0.353 & & & \\
\hline $5 \mathrm{H}-4,61$ & 38.82 & 13.833 & 0.596 & 0.80 & 0.476 & 0.426 & 0.443 & 0.398 & 0.028 & 0.0002 \\
\hline $5 \mathrm{H}-4,116$ & 39.37 & 13.925 & 0.597 & 0.82 & 0.489 & & 0.464 & & & \\
\hline $5 \mathrm{H}-5,18$ & 39.79 & 13.996 & 0.596 & 0.76 & 0.453 & 0.471 & 0.413 & 0.438 & 0.033 & 0.0001 \\
\hline $5 \mathrm{H}-5,61$ & 40.22 & 14.068 & 0.596 & 0.81 & 0.483 & & 0.460 & & & \\
\hline $5 \mathrm{H}-5,116$ & 40.77 & 14.160 & 0.596 & 0.79 & 0.471 & 0.477 & 0.433 & 0.446 & 0.031 & 0.0001 \\
\hline $5 \mathrm{H}-6, \mathrm{I} 8$ & 41.18 & 14.229 & 0.596 & 0.79 & 0.471 & & 0.442 & & & \\
\hline $5 \mathrm{H}-6,61$ & 41.61 & 14.301 & 0.596 & 0.75 & 0.447 & 0.459 & 0.416 & 0.429 & 0.030 & 0.0001 \\
\hline $6 \mathrm{H}-1,18$ & 44.68 & 15.025 & 0.397 & 0.71 & 0.282 & & 0.266 & & & \\
\hline
\end{tabular}


Table 9 (continued).

\begin{tabular}{|c|c|c|c|c|c|c|c|c|c|c|}
\hline $\begin{array}{l}\text { Core, section, } \\
\text { interval }(\mathrm{cm})\end{array}$ & $\begin{array}{l}\text { Depth } \\
\text { (mbsf) }\end{array}$ & $\begin{array}{l}\text { Age } \\
\text { (Ma) }\end{array}$ & $\begin{array}{c}\text { LSR } \\
(\mathrm{cm} / \mathrm{k} \cdot \mathrm{y} .)\end{array}$ & $\underset{\left(\mathrm{g} / \mathrm{cm}^{3}\right)}{\text { DBD }}$ & MAR & $\begin{array}{l}\text { MAR } \\
\text { (ave.) }\end{array}$ & CAR & $\begin{array}{l}\text { CAR } \\
\text { (ave.) }\end{array}$ & $\begin{array}{l}\text { NCAR } \\
\text { (ave.) }\end{array}$ & $\begin{array}{l}\text { EAR } \\
\text { (ave.) }\end{array}$ \\
\hline $6 \mathrm{H}-1,60$ & 45.10 & 15.130 & 0.397 & 0.78 & 0.310 & 0.305 & 0.291 & 0.288 & 0.018 & 0.0010 \\
\hline $6 \mathrm{H}-1,116$ & 45.66 & 15.271 & 0.397 & 0.61 & 0.242 & & 0.232 & & & \\
\hline $6 \mathrm{H}-2,18$ & 45.92 & 15.337 & 0.398 & 0.60 & 0.239 & 0.240 & 0.220 & 0.226 & 0.014 & 0.0007 \\
\hline $6 \mathrm{H}-2,60$ & 46.34 & 15.443 & 0.397 & 0.69 & 0.274 & & 0.257 & & & \\
\hline $6 \mathrm{H}-2,116$ & 46.90 & 15.584 & 0.397 & 0.68 & 0.270 & 0.272 & 0.253 & 0.255 & 0.017 & 0.0010 \\
\hline $6 \mathrm{H}-3,18$ & 47.33 & 15.692 & 0.397 & 0.69 & 0.274 & & 0.257 & & & \\
\hline $6 \mathrm{H}-3,60$ & 47.75 & 15.798 & 0.397 & 0.66 & 0.262 & 0.268 & 0.242 & 0.249 & 0.019 & 0.0002 \\
\hline $6 \mathrm{H}-3,116$ & 48.31 & 15.939 & 0.397 & 0.73 & 0.290 & & 0.274 & & & \\
\hline $6 \mathrm{H}-4,18$ & 48.71 & 16.040 & 0.397 & 0.76 & 0.302 & 0.296 & 0.284 & 0.279 & 0.017 & 0.0002 \\
\hline $6 \mathrm{H}-4,60$ & 49.13 & 16.145 & 0.397 & 0.77 & 0.306 & & 0.288 & & & \\
\hline $6 \mathrm{H}-4,116$ & 49.69 & 16.287 & 0.397 & 0.77 & 0.306 & 0.306 & 0.288 & 0.288 & 0.017 & 0.0004 \\
\hline $6 \mathrm{H}-5,18$ & 50.07 & 16.382 & 0.397 & 0.62 & 0.246 & & 0.234 & & & \\
\hline $6 \mathrm{H}-5,60$ & 50.49 & 16.488 & 0.397 & 0.62 & 0.246 & 0.246 & 0.231 & 0.233 & 0.014 & 0.0003 \\
\hline $6 \mathrm{H}-5,116$ & 51.05 & 16.629 & 0.397 & 0.72 & 0.286 & & 0.261 & & & \\
\hline $6 \mathrm{H}-6,18$ & 51.45 & 16.730 & 0.397 & 0.64 & 0.254 & 0.270 & 0.240 & 0.250 & 0.020 & 0.0003 \\
\hline
\end{tabular}

Notes: $\mathrm{LSR}=$ linear sedimentation rates $(\mathrm{cm} / \mathrm{k} . \mathrm{y}$.), $\mathrm{DBD}=$ dry bulk density, $\mathrm{MAR}=$ mass (bulk) sediment accumulation rate, $\mathrm{CAR}=$ carbonate accumulation rate, $\mathrm{NCAR}=$ noncarbonate accumulation rate, and $\mathrm{EAR}=$ eolian (e.g., fine-grained lithogenic material, $<63 \mu \mathrm{m}$ in size) accumulation rate. Accumulation rates are given in units of g/cm ${ }^{2} / \mathrm{k} . \mathrm{y}$. Average values are used when two successive samples were used to conduct the chemical extraction procedure. 